Article

\title{
Investigation of Novel Pesticides with Insecticidal and Antifungal Activities: Design, Synthesis and SAR Studies of Benzoylpyrimidinylurea Derivatives
}

\author{
Peiqi Chen ${ }^{1,2,3}$, Xiangmin Song ${ }^{1,2}$, Yongmei Fan ${ }^{1,2}$, Weihao Kong ${ }^{1,2}$, Hao Zhang ${ }^{3}$ \\ and Ranfeng Sun $1,2, *$ (D) \\ 1 Key Laboratory of Green Prevention and Control of Tropical Plant Diseases and Pests, Hainan University, \\ Haikou 570228, China; chenpq17@lzu.edu.cn (P.C.); 18898958572@163.com (X.S.); \\ yongmeifan@126.com (Y.F.); 15799032481@163.com (W.K.) \\ 2 Institute of Tropical Agriculture and Forestry, Hainan University, Haikou 570228, China \\ 3 School of Chemical \& Chemical Engineering, Inner Mongolia University, Hohhot 010021, China; \\ haozhang@imu.edu.cn \\ * Correspondence: srf18@hainu.edu.cn; Tel.: +86-898-6619-2906
}

Received: 9 August 2018; Accepted: 28 August 2018; Published: 31 August 2018

\begin{abstract}
In order to find pesticides with insecticidal and antifungal activities, a series of novel benzoyl pyrimidinylurea derivatives were designed and synthesized. All target compounds were identified by ${ }^{1} \mathrm{H}-\mathrm{NMR}$ spectroscopy and HRMS. Insecticidal and antifungal activity of these compounds were evaluated and the structure-activity relationships (SAR) were clearly and comprehensively illustrated. Compound 7, with low toxicity to zebrafish $\left(\mathrm{LC}_{50}=378.387 \mu \mathrm{g} \mathrm{mL}^{-1}\right)$ showed $100 \%$ inhibition against mosquito (Culex pipiens pallens) at $0.25 \mu \mathrm{g} \mathrm{mL}^{-1}$. Both compounds 19 and 25 exhibited broad-spectrum fungicidal activity ( $>50 \%$ inhibitory activities against 13 phytopathogenic fungi), which were better than those of the commercial pesticide pyrimethanil ( $>50 \%$ inhibitory activities against eight phytopathogenic fungi). Furthermore, compounds 19 and 25 exhibited protective activity against Sclerotinia sclerotiorum on leaves of Brassica oleracea L. during in vivo experiments.
\end{abstract}

Keywords: benzoylpyrimidinylurea derivatives; antifungal activity; insecticidal activity; embryo toxicity; zebrafish

\section{Introduction}

Plant disease and pest control are at least as old as agriculture because there is a need to keep crops free of pests and phythopatogenic fungi. Benzoylphenylureas (BPUs) have been developed as chitin synthesis inhibitors because of their unique mode of action coupled with a high degree of activity on targeted pests and low toxicity to non-target organisms [1,2]. The first commercial product Dimilin (diflubenzuron) (Figure 1), introduced in the market at 1975, exhibits broad spectrum larvicidal activities against caterpillars (Pieris brassicae), mosquitoes (Aedes aegypti), houseflies (Musca domestica), desert locust (Schistocera gregaria) and cotton strainers (Dysdercus superstitiosus) [3]. In the past few years, we and our co-workers have designed and synthesized several series of benzoylphenylureas (A and B, Figure 1) and most compounds exhibited excellent insecticidal activities against oriental armyworm (Mythimna separata) or mosquito (Culex pipiens pallens) [4-8]. Interestingly, compounds C and $\mathbf{D}$ (Figure 1) with a 2,2,2-trifluoroethyl group $\left(\mathrm{R}=\mathrm{CH}_{2} \mathrm{CF}_{3}\right)$ substituent, displayed much better insecticidal activities against oriental armyworm than other derivatives. 
<smiles>O=C(NC(=O)c1c(F)cccc1F)Nc1ccc(Cl)cc1</smiles>

Diflubenzuron<smiles>[R]C1ON=C(c2ccc(NC(=O)NC(=O)c3c(F)cccc3F)cc2)C1[R]</smiles>

B<smiles>[R]OS(=O)c1ccc(NC(=O)NC(=O)c2c(F)cccc2F)cc1</smiles><smiles>[R]C=C(C([R])=NO[R1])c1ccc(NC(=O)NC(=O)c2c(F)cccc2F)cc1</smiles>

A<smiles>[R]c1ccc(NC(=O)NC(=O)c2c(F)cccc2F)nn1</smiles>

C

Figure 1. Chemical structures of benzoylurea compounds.

At the same time, a class of phenylurea compounds (E, Figure 2) containing a pyrimidine ring attracted our attention. When bearing two 2,2,2-trifluoroethyl groups on the pyrimidine ring most of these compounds have good insecticidal activity performance against second-instar corn earworm (Helicoverpa zea) and beet armyworm (Spodoptera exigua) at $50 \mu \mathrm{g} \mathrm{mL}^{-1}$.<smiles>[R]c1ccc(NC(=O)Nc2nc(OCC(F)(F)F)cc(OCC(F)(F)F)n2)cc1</smiles>

$\mathbf{E}$<smiles>CC(=O)N1CCC(C2NC(c3ccc(F)cc3)C(c3ccnc(Nc4cccc(CO)c4)n3)S2)CC1</smiles><smiles>Cc1cc(C)nc(Nc2ccccc2)n1</smiles>

Pyrimethanil

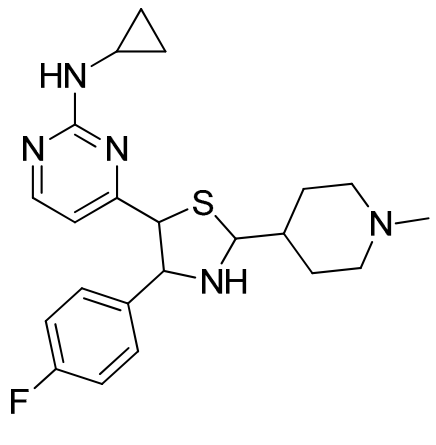

G

Figure 2. Chemical structures of compounds with pyrimidine groups 
More importantly, the 2-aminopyrimidinyl unit is commonly found in the chemical structure of antifungal compounds. For example, the antifungal activity of commercial pyrimethanil (Figure 2) against Alternaria kikuchiana Tanaka was $100 \%$ at $0.1 \mu \mathrm{g} \mathrm{mL}{ }^{-1}$ [9-12]. Compound $\mathbf{F}$ $\left(\mathrm{EC}_{50}=1.03 \mu \mathrm{g} \mathrm{mL}^{-1}\right)$ exhibited better antifungal activity against Phytophthora capsici than dimethomorph $\left(\mathrm{EC}_{50}=4.26 \mu \mathrm{g} \mathrm{mL}^{-1}\right.$ ) (Figure 2) [13]. Compound $\mathbf{G}$ (Figure 2) with excellent antifungal activity $\left(\mathrm{EC}_{50}=0.84 \mu \mathrm{g} \mathrm{mL}^{-1}\right)$ against Phytophthora capsici was discovered in 2011 by Nam et al. [14].

Based on our previous works [4-8] and the literature [9-14], in order to find new pesticides with powerful insecticidal and antifungal properties, we were interested in introducing pyrimidine group into the benzoylurea backbone to design a series of benzoylpyrimidinylurea derivatives (Figure 3). Therefor target compounds 1-38 were synthesized and their insecticidal and antifungal activities were evaluated. At the same time, the aquatic toxicity of compounds $\mathbf{7}$ and $\mathbf{2 5}$ were evaluated by a zebrafish embryo acute toxicity test (OECD TG 236).<smiles>O=C(Nc1ccc(S(=O)OCC(F)(F)F)cc1)Nc1ccc(OCC(F)(F)F)nn1</smiles><smiles>Cc1cc(C)nc(Nc2ccccc2)n1</smiles><smiles>O=C(Nc1c#[R]ccc1)Nc1nc(OCC(F)(F)F)cc(OCC(F)(F)F)n1</smiles>

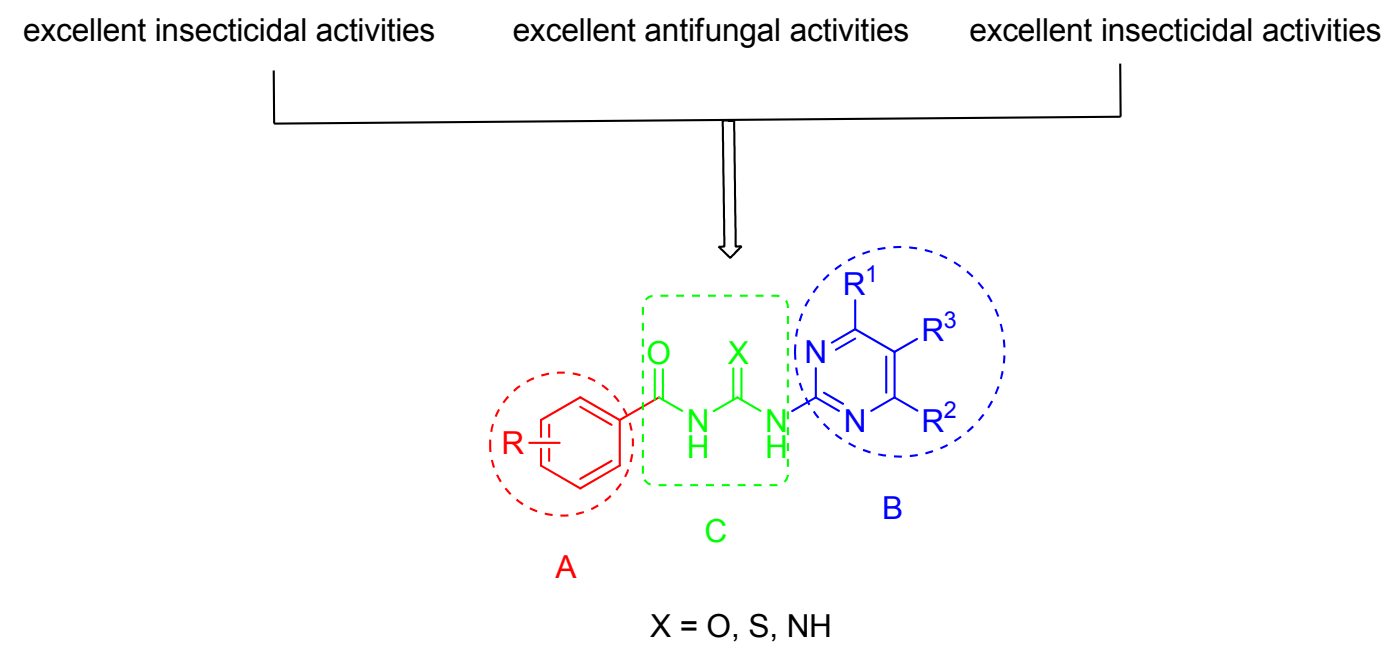

Figure 3. Design strategies of the target benzoylpyrimidinylurea derivatives.

\section{Results}

\subsection{Synthesis}

In order to comprehensively analyze the structure-activity relationship of compounds, the chemical structures of the benzoylpyrimidinylureas was separated into three units, namely, the benzoyl ring (A), pyrimidine ring (B) and urea bridge (C) (Figure 3). Substituted 2-aminopyrimidines Ia-p were synthesized and used as starting materials for the synthesis of the target compounds (Schemes 1 and 2). 


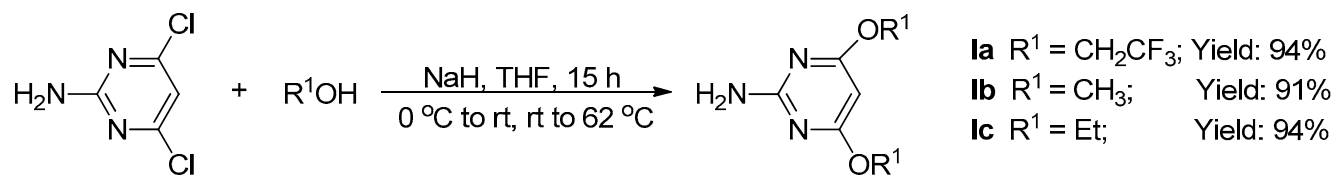

la-c

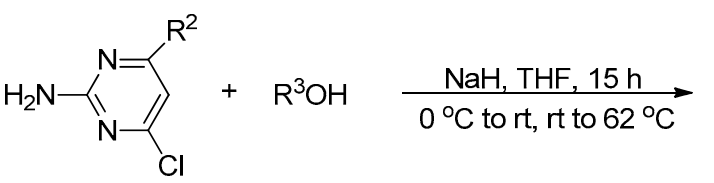<smiles>[R10]Oc1cc([R])nc(N)n1</smiles>

Id $\mathrm{R}^{2}=\mathrm{Cl} ; \mathrm{R}^{3}=\mathrm{CH}_{3} ; \quad$ Yield: $84 \%$ le $\mathrm{R}^{2}=\mathrm{CH}_{3} ; \mathrm{R}^{3}=\mathrm{CH}_{2} \mathrm{CF}_{3}$; Yield: $69 \%$ If $\mathrm{R}^{2}=\mathrm{Cl} ; \mathrm{R}^{3}=\mathrm{CH}_{2} \mathrm{CF}_{3} ; \quad$ Yield: $76 \%$

\section{Id-f}

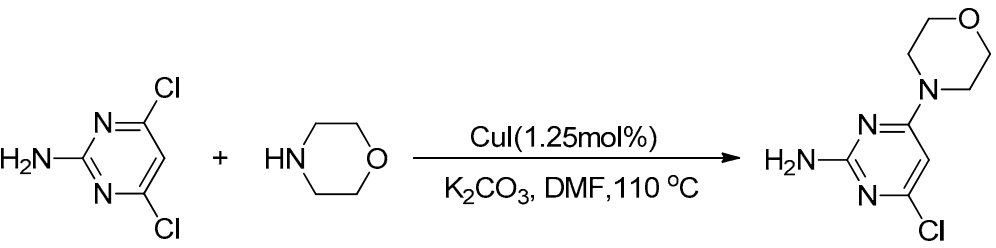

Ig

Yield: $79 \%$

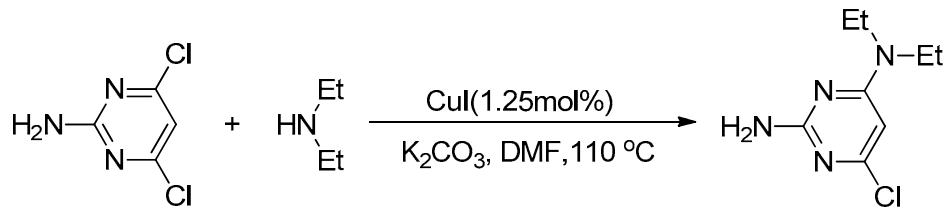

Ih

Yield: $81 \%$<smiles>COO[C@H](OC(C)=O)C1CCCN1</smiles>

If

li

Yield: $51 \%$

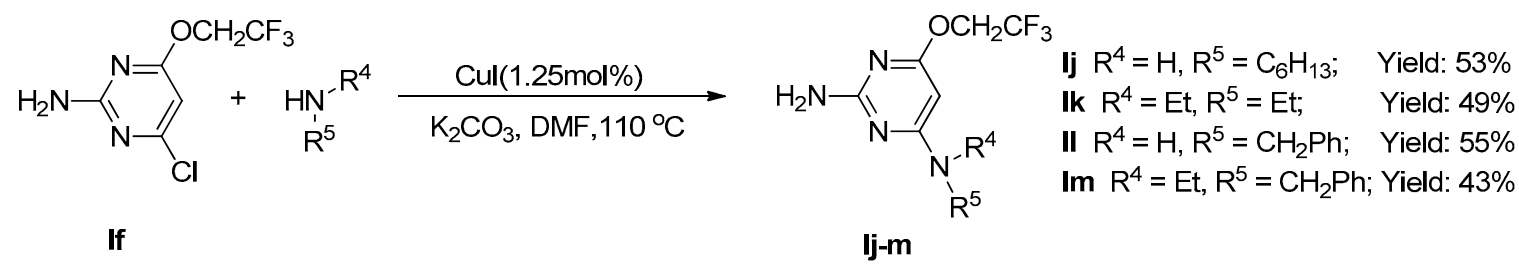

Scheme 1. Synthetic route for substituted 2-aminopyrimidines Ia-c, Id-f and Ig-m. 
<smiles>CCOC(=O)C(C(=O)OCC)C(C)C(C)CC</smiles><smiles>CCOC(C)C(C)C(Cl)Cl</smiles>

In

Yield: $73 \%$<smiles>CCNCCC(C)C(C)C(C)CC</smiles>

In<smiles>CCC(C)c1c(Cl)nc(N)nc1Cl</smiles>

In<smiles>CCC(C)c1c(Cl)nc(N)nc1OCC(F)(F)F</smiles>

Ip

Yield: $40 \%$

Scheme 2. Synthetic route for substituted 2-aminopyrimidines In, Io and Ip.

In a first series of compounds, intermediate Ia was retained and the benzoyl moiety was altered resulting in compounds $\mathbf{1}$ to $\mathbf{1 0}$ (Scheme 3).
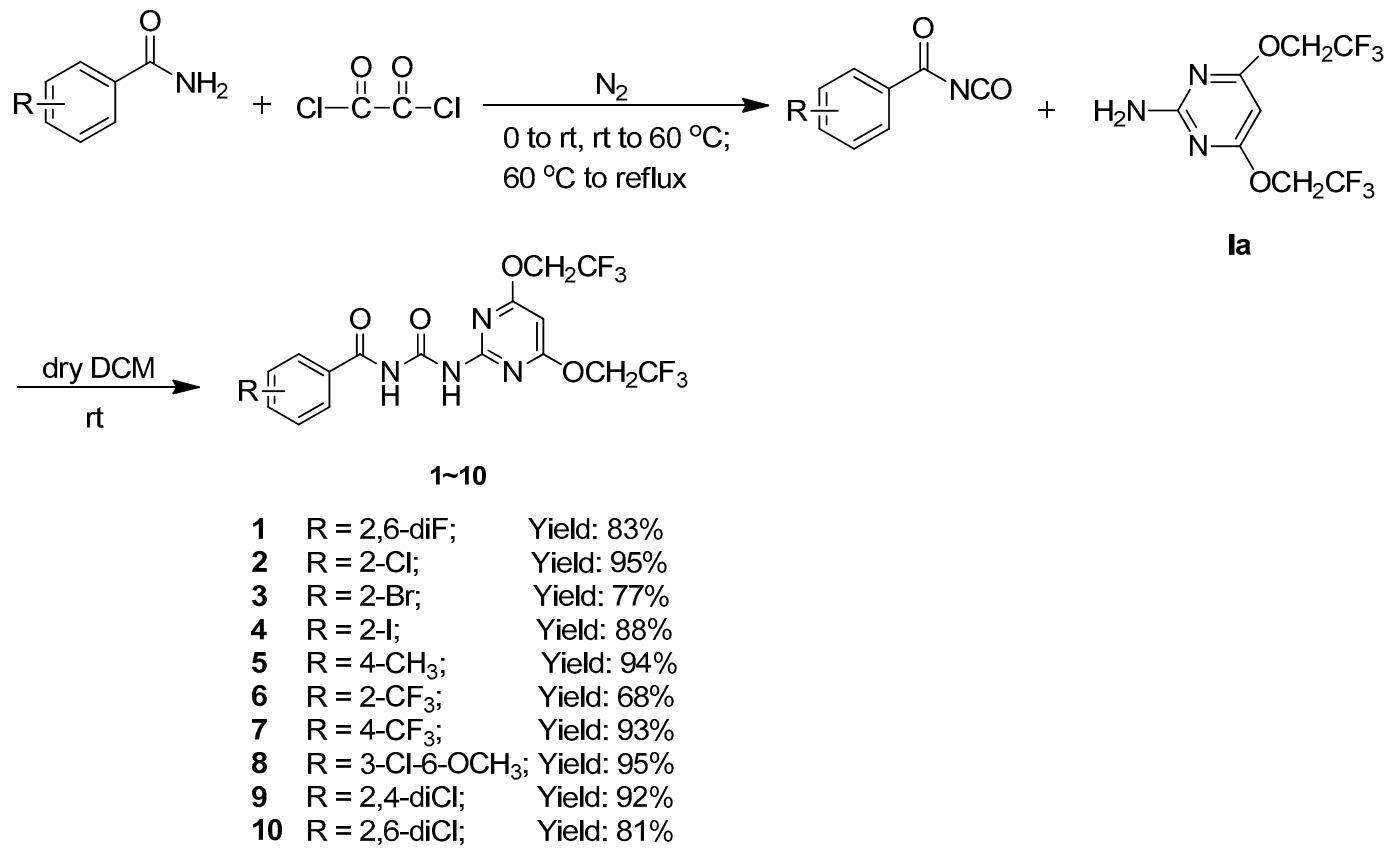

la

Scheme 3. Synthetic route for compounds 1-10. 
Secondly, different substituents were changed on the pyrimidine ring and the compounds 11-30 with substituted 2-aminopyrimidines were obtained (Scheme 4).<smiles>[X]c1nc(N)nc(N)n1</smiles>
$60^{\circ} \mathrm{C}$ to reflux

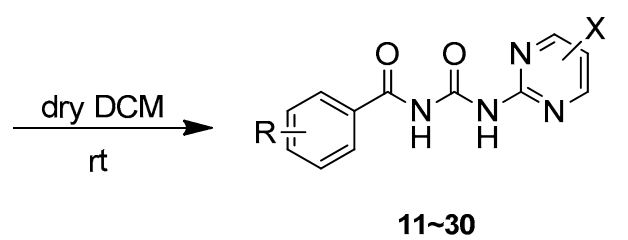

$11 \mathrm{R}=\mathrm{Cl} ; \mathrm{X}=4,6-\mathrm{diCH}_{3}$;

$12 \mathrm{R}=\mathrm{Cl} ; \mathrm{X}=4,6-\mathrm{diOCH}_{3}$;

Yield: $87 \%$;

$13 \mathrm{R}=\mathrm{Cl} ; \mathrm{X}=4,6$-diOEt;

Yield: $89 \%$;

$14 \mathrm{R}=\mathrm{Cl} ; \mathrm{X}=4,6-\mathrm{diCl}$;

$15 \mathrm{R}=\mathrm{Cl} ; \mathrm{X}=4-\mathrm{Cl}-6-\mathrm{CH}_{3}$;

Yield: $91 \%$;

$16 \mathrm{R}=\mathrm{Cl} ; \mathrm{X}=4-\mathrm{Cl}-6-\mathrm{OCH}_{3}$;

Yield: $88 \%$;

Yield: $93 \%$;

$17 \mathrm{R}=\mathrm{Cl} ; \mathrm{X}=4-\mathrm{Cl}-6-\mathrm{OCH}_{2} \mathrm{CF}_{3}$;

$18 \mathrm{R}=\mathrm{Cl} ; \mathrm{X}=4-\mathrm{Cl}-6$-morpholinyl;

Yield: $87 \%$;

Yield: $88 \%$;

Yield: $85 \%$;

$19 \mathrm{R}=\mathrm{Cl} ; \mathrm{X}=4-\mathrm{Cl}-6$-diethylamino;

Yield: $83 \%$;

$20 \mathrm{R}=\mathrm{Cl} ; \mathrm{X}=4-\mathrm{CH}_{3}-6-\mathrm{OCH}_{2} \mathrm{CF}_{3} ; \quad$ Yield: $82 \%$;

$21 \mathrm{R}=\mathrm{Cl} ; \mathrm{X}=4$-(pyrrolidin-1-yl)-6- $\mathrm{OCH}_{2} \mathrm{CF}_{3}$; Yield: $81 \%$;

$22 \mathrm{R}=\mathrm{Cl} ; \mathrm{X}=4$-hexylamino-6- $\mathrm{OCH}_{2} \mathrm{CF}_{3}$; Yield: $83 \%$;

$23 \mathrm{R}=2,4$-diCl; $\mathrm{X}=4,6-\mathrm{diCH}_{3}$; $\quad$ Yield: $81 \%$;

$24 \mathrm{R}=2,4-$ diCl; $\mathrm{X}=4-\mathrm{CH}_{3}-6-\mathrm{OCH}_{2} \mathrm{CF}_{3} ; \quad$ Yield: $81 \%$;

$25 \mathrm{R}=2,6-$ diCl; $\mathrm{X}=4-\mathrm{Cl}-6-\mathrm{OCH}_{2} \mathrm{C}_{3}$; $\quad$ Yield: $87 \%$;

$26 \mathrm{R}=2$,6-diCl; $\mathrm{X}=4$ 4-Cl-6-diethylamino; $\quad$ Yield: 81\%;

$27 \mathrm{R}=2,6-$ diCl; $\mathrm{X}=4$ 4-diethylamino-6- $\mathrm{OCH}_{2} \mathrm{CF}_{3} ; \quad$ Yield: $87 \%$;

$28 \mathrm{R}=2,6$-diCl; $\mathrm{X}=4$ 4-benzylamino-6- $\mathrm{OCH}_{2} \mathrm{CF}_{3} ; \quad$ Yield: $80 \%$;

$29 \mathrm{R}=2,6-$ diCl; $\mathrm{X}=4$-(benzyl(ethyl)amino-6- $\mathrm{OCH}_{2} \mathrm{CF}_{3}$; Yield: $75 \%$;

$30 \mathrm{R}=2,6-$ diCl; $\mathrm{X}=5$-(sec-butyl)4-Cl-6- $\mathrm{OCH}_{2} \mathrm{CF}_{3} ; \quad$ Yield: $73 \%$;

Scheme 4. Synthetic route for compounds 11-30.

Thirdly, we replaced the oxygen atom on the urea bridge with sulfur or nitrogen to gain compounds 31 and 32 (Scheme 5). 


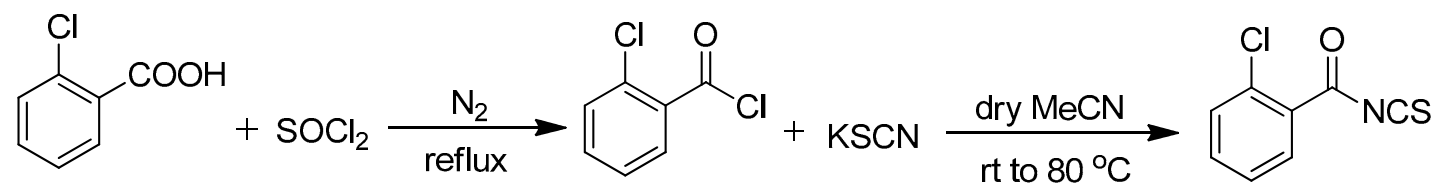<smiles>Nc1nc(OCC(F)(F)F)cc(OCC(F)(F)F)n1</smiles>

la

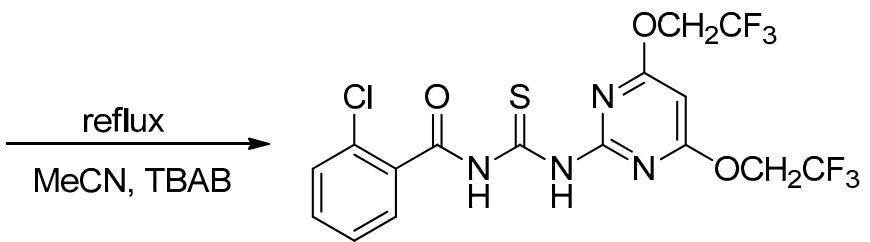

31

Yield: 53\%

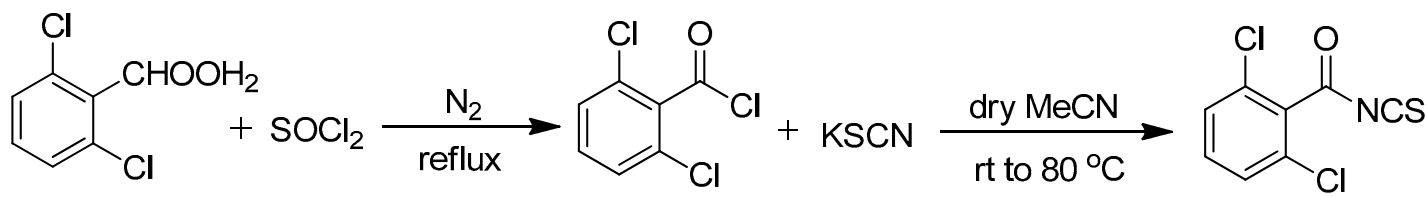<smiles>CCN(CC)c1cc(OCC(F)(F)F)nc(N)n1</smiles>

Ik<smiles>CCN(CC)c1cc(OCC(F)(F)F)nc(NC(=S)NC(=O)c2c(Cl)ccc(CC(F)(F)Br)c2Cl)n1</smiles>

32a

Yield: $59 \%$<smiles>CCN(CC)c1cc(OCC(F)(F)F)nc(NC(=N)NC(=O)c2c(Cl)cccc2Cl)n1</smiles>

32

Yield: $81 \%$

Scheme 5. Synthetic route for compounds 31 and 32.

Finally, the benzoyl ring was exchanged by a six- or five-membered nitrogen heterocycles to compare the effects between benzene ring and heterocycles on the bioactivity of the compounds, therefor compounds 33-37 were synthesized (Scheme 6). To illustrate the role of the carbonyl group of the benzoyl group in the biological activity of the target compounds, compound $\mathbf{3 8}$ was synthesized from 2,6-dichloroaniline (Scheme 6). All reactions gave excellent yields and the NMR data of compounds proved the proposed structures. Among them, compounds with trifluoromethyl groups have a characteristic quartet peak with ${ }^{13} \mathrm{C}-{ }^{19} \mathrm{~F}$ couplings in the NMR spectra. The spectra are shown in Figures S1-S101 in the Supporting Information. 


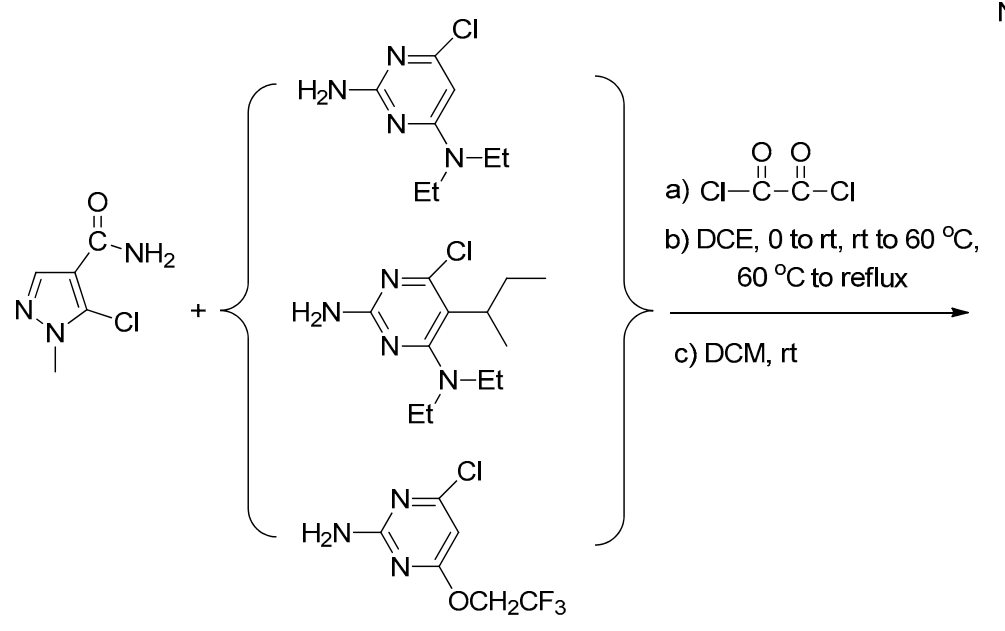<smiles>CCC(C)c1c(Cl)nc(N)nc1Cl</smiles><smiles>CCN(CC)c1cc(Cl)nc(NC(=O)NC(=O)c2cnn(C)c2Cl)n1</smiles><smiles>[R16][R16]#[Y9]</smiles>

a) $\stackrel{\mathrm{Ol}}{\mathrm{Cl}} \stackrel{\mathrm{O}}{\mathrm{C}}-\stackrel{\mathrm{C}}{\mathrm{C}}-\mathrm{Cl}$<smiles>CCN(CC)c1cc(Cl)nc(N)n1</smiles>

b) DCE, 0 to $r$, it to $60^{\circ} \mathrm{C}$, $60^{\circ} \mathrm{C}$ to reflux c) DCM, rt<smiles>Clc1cccc(Cl)c1I</smiles><smiles>CCN(CC)c1cc(Cl)nc(NC(N)=O)n1</smiles>

Scheme 6. Synthetic route for compounds 33-38.

\subsection{Bioassays}

\subsubsection{Toxicity against Mosquito (Culex pipiens pallens)}

Table 1 shows the larvicidal activities of the compounds 1-25, 31, 37 and fipronil against mosquito. The bioassay results indicated that eighteen compounds (1-7, 9-10, 13-14, 16, 18, 20, 22-24, 31) exhibited more than 50\% larvicidal activities against mosquito and eleven of them reached 
$100 \%$ at $10 \mu \mathrm{g} \mathrm{mL}^{-1}$, especially, compound 7 which showed $100 \%$ mortality even at $0.25 \mu \mathrm{g} \mathrm{mL}{ }^{-1}$. By comparing the chemical structure of compounds $2(2-\mathrm{Cl}), 3(2-\mathrm{Br})$ and $4(2-\mathrm{I})$, we found that the larvicidal activitives decreased $\left(100 \%, 60 \%\right.$ mortality at $2 \mu \mathrm{g} \mathrm{mL} \mathrm{m}^{-1}, 50 \%$ mortality at $10 \mu \mathrm{g} \mathrm{mL}$ respectively) as the radius of the halogen atom at the 2-position on the benzoyl ring increased. Next, we found that when the 2,6-position on the benzoyl ring was substituted by two halogens or electron-rich groups, it was not conducive to larvicidal activity, for example, the larva mortalities of compounds $8\left(2-\mathrm{OCH}_{3}\right), 9(2,4-\mathrm{di}-\mathrm{Cl})$ and $10(2,6-\mathrm{di}-\mathrm{Cl})$ were $20 \%$ at $10 \mu \mathrm{g} \mathrm{mL}-1,40 \%$ at $2 \mu \mathrm{g} \mathrm{mL}{ }^{-1}$ and $20 \%$ at $5 \mu \mathrm{g} \mathrm{mL}^{-1}$ respectively.

Table 1. Larvicidal Activities against Oriental Armyworm and Mosquito of Compounds 1-25, 31, 37.

\begin{tabular}{|c|c|c|c|c|c|c|c|c|c|c|}
\hline \multirow{3}{*}{ Compd. } & \multicolumn{10}{|c|}{ Larvicidal Activity (\%) } \\
\hline & \multicolumn{3}{|c|}{ Oriental Armyworm $\left(\mu \mathrm{g} \mathrm{mL}^{-1}\right)$} & \multicolumn{7}{|c|}{ Mosquito ( $\mu \mathrm{g} \mathrm{mL}^{-1}$ ) } \\
\hline & 600 & 200 & 100 & 10 & 5 & 2 & 1 & 0.5 & 0.25 & 0.1 \\
\hline 1 & 45 & $-a$ & - & 100 & 100 & 60 & - & - & - & - \\
\hline 2 & 5 & - & - & 100 & 100 & 100 & 100 & 100 & 20 & - \\
\hline 3 & 100 & 100 & 60 & 100 & 100 & 60 & - & - & - & - \\
\hline 4 & 70 & - & - & 50 & - & - & - & - & - & - \\
\hline 5 & 65 & - & - & 50 & - & - & - & - & - & - \\
\hline 6 & 15 & - & - & 100 & 100 & 20 & - & - & - & - \\
\hline 7 & 65 & - & - & 100 & 100 & 100 & 100 & 100 & 100 & 20 \\
\hline 8 & 10 & - & - & 20 & - & - & - & - & - & - \\
\hline 9 & 100 & 100 & 40 & 100 & 100 & 40 & - & - & - & - \\
\hline 10 & 40 & - & - & 100 & 20 & - & - & - & - & - \\
\hline 11 & 20 & - & - & 10 & - & - & - & - & - & - \\
\hline 12 & 5 & - & - & 20 & - & - & - & - & - & - \\
\hline 13 & 60 & - & - & 100 & 100 & 70 & - & - & - & - \\
\hline 14 & 50 & - & - & 100 & 100 & 60 & - & - & - & - \\
\hline 15 & 30 & - & - & 20 & - & - & - & - & - & - \\
\hline 16 & 5 & - & - & 100 & 0 & - & - & - & - & - \\
\hline 17 & 30 & - & - & 5 & - & - & - & - & - & - \\
\hline 18 & 35 & - & - & 70 & - & - & - & - & - & - \\
\hline 19 & 30 & - & - & 10 & - & - & - & - & - & - \\
\hline 20 & 10 & - & - & 80 & - & - & - & - & - & - \\
\hline 21 & 20 & - & - & 5 & - & - & - & - & - & - \\
\hline 22 & 30 & - & - & 65 & - & - & - & - & - & - \\
\hline 23 & 15 & - & - & 60 & - & - & - & - & - & - \\
\hline 24 & 20 & - & - & 65 & - & - & - & - & - & - \\
\hline 25 & 40 & - & - & 30 & - & - & - & - & - & - \\
\hline 31 & 100 & 20 & - & 100 & 100 & 100 & 50 & - & - & - \\
\hline 37 & 20 & - & - & 15 & - & - & - & - & - & - \\
\hline Fipronil & - & - & - & 100 & 100 & 100 & 100 & 100 & 100 & 100 \\
\hline
\end{tabular}

However, electron-withdrawing groups in the para position enhanced the bioactivity of the compounds, for example, the larva mortality of compound $7\left(4-\mathrm{CF}_{3}\right)$ reached $100 \%$ at $0.25 \mu \mathrm{g} \mathrm{mL}^{-1}$. When the 4,6-position on the pyrimidine ring were substituted with haloalkoxyl, alkoxy or alkyl groups, the larvicidal activity decreased successively, at the same time the longer the alkoxy chain, the larvicidal activity was better. For example, the mortalities of compounds $\mathbf{1 1}\left(4,6-\mathrm{di}-\mathrm{CH}_{3}\right), \mathbf{1 2}\left(4,6-\mathrm{di}-\mathrm{OCH}_{3}\right), \mathbf{1 3}$ (4,6-di-OEt) and 2 (4,6-di-OCH $\left.{ }_{2} \mathrm{CF}_{3}\right)$ were $10 \%$ at $10 \mu \mathrm{g} \mathrm{mL}^{-1}, 20 \%$ at $10 \mu \mathrm{g} \mathrm{mL}-1,70 \%$ at $2 \mu \mathrm{g} \mathrm{mL}^{-1}$, $100 \%$ at $0.5 \mu \mathrm{g} \mathrm{mL} \mathrm{L}^{-1}$, respectively. Similarly, the structure-activity relationships of compounds 2 and $\mathbf{1 4}$ told us that a trifluoroethoxy group was more helpful to increase the mortality activities than s halogen group. Furthormore it was easy to find that the urea bridge unit was important to the larvicidal activity. For example compound 31 containing a thiourea unit $\left(50 \%\right.$ mortality at $1 \mu \mathrm{g} \mathrm{mL} \mathrm{L}^{-1}$ ) exhibited lower larvicidal activity than compound $2\left(100 \%\right.$ mortality at $\left.0.5 \mu \mathrm{g} \mathrm{mL}^{-1}\right)$. Finally, when a pyridine ring 
(compound 37) was employed instead of a benzene ring (compound 2), the larvicidal activities were reduced greatly (15\% mortality of compound 37 at $10 \mu \mathrm{g} \mathrm{mL}^{-1}$ and $100 \%$ mortality of compound 2 at $0.5 \mu \mathrm{g} \mathrm{mL}^{-1}$ ). Therefore, it was concluded that electron-withdrawing groups (such as a trifuloromethyl group) on the para position of the benzoyl ring, alkoxy groups (especially haloalkoxyl groups, such as a trifluoroethoxy group) and a urea bridge unit favored the larvicidal activities.

\subsubsection{Stomach Toxicity against Oriental Armyworm (Mythimna separata)}

The bioactivity results indicated that most compounds exhibited certain insecticidal activities against oriental armyworm as listed in Table 1. Firstly, we can easily see from compounds 2 (2-Cl) and $5(4-\mathrm{Cl})$ or $6\left(2-\mathrm{CF}_{3}\right)$ and $7\left(4-\mathrm{CF}_{3}\right)$ and their larva moralities $\left(5 \%, 65 \%, 15 \%\right.$ and $65 \%$ at $600 \mu \mathrm{g} \mathrm{mL}{ }^{-1}$ respectively) that para-substituents of the benzoyl ring result in better insecticidal activities than ortho-substituents. Secondly, it was figured out that both the radius and electronegativity of the halogen atom at the 2-position on the benzoyl ring influenced the larvicidal activities, for example, compound $3\left(2-\mathrm{Br}, 100 \%\right.$ mortality at $\left.200 \mu \mathrm{g} \mathrm{mL}{ }^{-1}\right)$ was a more effective insecticidal agent than compound 4 (2-I, 70\% mortality at $\left.600 \mu \mathrm{g} \mathrm{mL}^{-1}\right)$ which exhibited much better larvicidal activity than compound $2\left(2-\mathrm{Cl}, 5 \%\right.$ mortality at $\left.600 \mu \mathrm{g} \mathrm{mL}^{-1}\right)$. At the same time, it also pointed out that chlorine atom on the pyrimidine ring was beneficial to improve the insecticidal activity of compounds, for example, the larva mortalities of compounds $14(4,6-\mathrm{Cl}), 15\left(4-\mathrm{Cl}, 6-\mathrm{CH}_{3}\right)$ and $16\left(4-\mathrm{Cl}, 6-\mathrm{OCH}_{3}\right)$ were $50 \%, 30 \%, 5 \%$ at $600 \mu \mathrm{g} \mathrm{mL}^{-1}$ respectively. Interestingly, when we replaced the oxygen atom on the urea bridge with a sulfur atom, the insecticidal activities of the compounds increased greatly, as seen by comparing the insecticidal activities of compound $2\left(5 \%\right.$ mortality of at $\left.600 \mu \mathrm{g} \mathrm{mL} \mathrm{L}^{-1}\right)$ and compound $31\left(100 \%\right.$ mortality at $\left.600 \mu \mathrm{g} \mathrm{mL}^{-1}\right)$.

\subsubsection{In Vitro Antifungal Activity}

The fungicidal results are listed in Table 2. Most of the derivatives showed antifungal activity against 14 kinds of phytopathogenic fungi. We first determined the antifungal activities of compounds 2 (2-Cl), $3(2-\mathrm{Br}), 4(2-\mathrm{I}), 5(4-\mathrm{Cl}), \mathbf{6}\left(2-\mathrm{CF}_{3}\right), 7\left(4-\mathrm{CF}_{3}\right)$ and $8\left(2-\mathrm{OCH}_{3}, 5-\mathrm{Cl}\right)$ and we found that ortho-halogen substitutents on the benzoyl ring were beneficial for antifungal activity. Among them, compound 2 was better than the others. At this moment, our design strategy was divided into two parts. One was to maintain 2-chloro group on the benzoyl ring and a series of differently substituted compounds (such as compounds 11-22) at the 4,6-position on the pyrimidine ring were synthesized. The other was that we continued to explore the effect of the number of halogen atoms on the benzoyl ring for the antifungal activity, followed by the synthesis of compounds with different substitutions at the 4,6-position on pyrimidine ring, such as compounds 1, 9, 10 and 23-30.

From the first part of strategy, we reached three important conclusions. Firstly, compounds with alkoxy groups had better antifungal activity than those bearing an alkyl group, and if the hydrogen atom on the alkoxy group was substituted with a halogen atom, the antifungal activity of compounds would be further enhanced. For example, the antifungal activity order of compounds 2, 11, 12 and 13 was compound $2\left(4,6-\mathrm{di}-\mathrm{OCH}_{2} \mathrm{CF}_{3}\right)>$ compound $13(4,6$-di-OEt $)>$ compound $12\left(4,6-\mathrm{di}-\mathrm{OCH}_{3}\right)>$ compound 11 (4,6-di- $\left.\mathrm{CH}_{3}\right)$ (Table 2). Secondly, chlorine atoms were beneficial to increase the antifungal activity of the compounds. For example, the antifungal activity order of compounds 14, 15 and 16 was compound $14(4,6-\mathrm{Cl})>$ compound $15\left(4-\mathrm{Cl}, 6-\mathrm{CH}_{3}\right)>$ compound $16\left(4-\mathrm{Cl}, 6-\mathrm{OCH}_{3}\right)($ Table 2$)$. 
Table 2. Fungicidal Activity of Compounds 1-38 against Phytopathogenic Fungi at $50 \mu \mathrm{g} \mathrm{mL}{ }^{-1}$.

\begin{tabular}{|c|c|c|c|c|c|c|c|c|c|c|c|c|c|c|}
\hline \multirow{2}{*}{ Compd. } & \multicolumn{14}{|c|}{ Inhibition Rate (\%) } \\
\hline & $A . S^{\mathrm{a}}$ & F.G & $P . I$ & $P . C$ & $S . S$ & B.C & R.S & F.C & C.H & $P . P$ & R.C & B.M & $W . A$ & F.M \\
\hline 1 & 16.7 & 3.4 & 5.9 & 17.2 & 10.7 & 7.7 & 6.2 & 11.6 & 16.7 & 26.8 & 11.6 & 16.3 & 12.5 & 27.3 \\
\hline 2 & 16.7 & 44.8 & 35.3 & 41.9 & 62.5 & 34.6 & 35.8 & 41.9 & 46.7 & 91.1 & 89.5 & 55.1 & 47.5 & 57.6 \\
\hline 3 & 55.6 & 56.0 & 57.1 & 36.8 & 53.5 & 44.8 & 38.5 & 53.6 & 47.8 & 62.8 & 94.9 & 63.3 & 55.6 & 50.0 \\
\hline 4 & 44.4 & 68.0 & 57.1 & 31.6 & 41.9 & 37.9 & 30.8 & 50.0 & 47.8 & 81.4 & 93.2 & 76.7 & 63.0 & 45.5 \\
\hline 5 & 33.3 & 16.0 & 7.1 & 15.8 & 10.5 & 24.1 & 11.5 & 21.4 & 21.7 & 41.9 & 69.5 & 16.7 & 22.2 & 13.6 \\
\hline 6 & 16.7 & 37.9 & 23.5 & 38.7 & 44.6 & 34.6 & 35.8 & 34.9 & 43.3 & 57.1 & 83.7 & 51.0 & 45.0 & 48.5 \\
\hline 7 & 12.5 & 10.3 & 23.5 & 25.8 & 10.7 & 11.5 & 12.3 & 16.3 & 30.0 & 44.6 & 23.3 & 16.3 & 22.5 & 21.2 \\
\hline 8 & 16.7 & 41.4 & 17.6 & 32.3 & 14.3 & 11.5 & 6.2 & 14.0 & 16.7 & 26.8 & 17.4 & 14.3 & 27.5 & 18.2 \\
\hline 9 & 33.3 & 40.0 & 28.6 & 31.6 & 53.5 & 27.6 & 32.7 & 39.3 & 47.8 & 76.7 & 88.1 & 70.0 & 37.0 & 50.0 \\
\hline 10 & 55.6 & 56.0 & 50.0 & 31.6 & 58.1 & 51.7 & 32.7 & 57.1 & 52.2 & 58.1 & 94.9 & 66.7 & 51.9 & 68.2 \\
\hline 11 & 44.4 & 24.0 & 10.7 & 10.5 & 11.6 & 27.6 & 17.3 & 21.4 & 17.4 & 51.2 & 67.8 & 20.0 & 25.9 & 13.6 \\
\hline 12 & 44.4 & 36.0 & 14.3 & 26.3 & 18.6 & 13.8 & 9.6 & 25.0 & 30.4 & 46.5 & 62.7 & 33.3 & 33.3 & 13.6 \\
\hline 13 & 38.9 & 28.0 & 28.6 & 26.3 & 16.3 & 27.6 & 19.2 & 25.0 & 30.4 & 97.7 & 69.5 & 40.0 & 48.1 & 22.7 \\
\hline 14 & 44.4 & 24.0 & 28.6 & 31.6 & 20.9 & 58.6 & 7.7 & 14.3 & 43.5 & 60.5 & 71.2 & 30.0 & 22.2 & 18.2 \\
\hline 15 & 33.3 & 36.0 & 21.4 & 68.4 & 16.3 & 44.8 & 11.5 & 10.7 & 17.4 & 51.2 & 67.8 & 20.0 & 25.9 & 13.6 \\
\hline 16 & 27.8 & 20.0 & 21.4 & 26.3 & 14.0 & 10.3 & 11.5 & 21.4 & 17.4 & 55.8 & 67.8 & 20.0 & 33.3 & 18.2 \\
\hline 17 & 38.9 & 16.0 & 21.4 & 26.3 & 10.5 & 20.7 & 21.2 & 17.9 & 30.4 & 32.6 & 47.5 & 20.0 & 33.3 & 13.6 \\
\hline 18 & 33.3 & 32.0 & 7.1 & 15.8 & 11.6 & 20.7 & 9.6 & 14.3 & 30.4 & 51.2 & 62.7 & 20.0 & 33.3 & 22.7 \\
\hline 19 & 66.7 & 64.0 & 64.3 & 73.7 & 83.7 & 75.9 & 46.2 & 60.7 & 60.9 & 88.0 & 93.2 & 70.0 & 66.7 & 63.6 \\
\hline 20 & 55.6 & 36.0 & 42.9 & 26.3 & 14.0 & 10.3 & 11.5 & 39.3 & 47.8 & 76.7 & 84.7 & 66.7 & 59.3 & 50.0 \\
\hline 21 & 33.3 & 31.6 & 36.4 & 27.8 & 19.6 & 47.6 & 16.0 & 37.1 & 40.0 & 44.9 & 87.3 & 50.0 & 44.4 & 50.0 \\
\hline 22 & 22.2 & 31.6 & 18.2 & 19.4 & 53.6 & 23.8 & 33.3 & 37.1 & 30.0 & 51.0 & 87.3 & 44.4 & 29.6 & 46.2 \\
\hline 23 & 27.8 & 12.0 & 14.3 & 26.3 & 10.5 & 10.3 & 9.6 & 7.1 & 26.1 & 67.4 & 59.3 & 3.3 & 37.0 & 18.2 \\
\hline 24 & 11.1 & 12.0 & 10.7 & 26.3 & 11.6 & 17.2 & 9.6 & 25.0 & 30.4 & 67.4 & 71.2 & 13.3 & 14.8 & 9.1 \\
\hline 25 & 55.6 & 44.0 & 57.1 & 52.6 & 90.7 & 89.7 & 57.7 & 60.7 & 69.6 & 84.2 & 94.9 & 66.7 & 59.3 & 63.6 \\
\hline 26 & 44.4 & 31.6 & 54.5 & 61.1 & 82.1 & 47.6 & 30.9 & 48.6 & 60.0 & 51.0 & 92.7 & 55.6 & 48.1 & 53.8 \\
\hline 27 & 22.2 & 31.6 & 27.3 & 19.4 & 26.8 & 23.8 & 18.5 & 34.3 & 35.0 & 65.3 & 89.1 & 47.2 & 40.7 & 53.8 \\
\hline 28 & 16.7 & 18.4 & 27.3 & 27.8 & 53.6 & 14.3 & 6.2 & 14.3 & 5.0 & 6.1 & 58.2 & 22.2 & 14.8 & 26.9 \\
\hline 29 & 11.1 & 5.3 & 4.5 & 27.8 & 17.9 & 19.0 & 6.2 & 31.4 & 25.0 & 26.5 & 72.7 & 27.8 & 14.8 & 30.8 \\
\hline 30 & 31.3 & 45.5 & 21.1 & 27.3 & 33.3 & 12.5 & 37.9 & 16.7 & 45.5 & 56.4 & 66.2 & 28.9 & 21.4 & 27.3 \\
\hline 31 & 8.3 & 15.8 & 20.7 & 24.0 & 5.4 & 31.0 & 6.6 & 8.3 & 9.5 & 13.2 & 27.3 & 29.6 & 7.4 & 17.9 \\
\hline 32 & 11.1 & 21.1 & 27.3 & 22.2 & 23.2 & 14.3 & 6.2 & 17.1 & 15.0 & 8.2 & 56.4 & 19.4 & 18.5 & 30.8 \\
\hline 33 & 31.3 & 27.3 & 31.6 & 40.9 & 29.4 & 18.8 & 22.7 & 36.7 & 50.0 & 89.7 & 78.4 & 44.7 & 21.4 & 40.9 \\
\hline 34 & 43.8 & 31.8 & 15.8 & 4.5 & 33.3 & 18.8 & 18.2 & 10.0 & 27.3 & 66.7 & 20.3 & 31.6 & 14.3 & 18.2 \\
\hline 35 & 12.5 & 27.3 & 15.8 & 4.5 & 11.8 & 18.8 & 12.1 & 6.7 & 18.2 & 46.2 & 37.8 & 15.8 & 10.7 & 18.2 \\
\hline 36 & 37.5 & 36.4 & 31.6 & 27.3 & 49.0 & 31.3 & 30.3 & 56.7 & 50.0 & 79.5 & 78.4 & 68.4 & 57.1 & 54.5 \\
\hline 37 & 44.4 & 44.7 & 50.0 & 55.6 & 81.7 & 47.6 & 34.6 & 54.3 & 40.0 & 22.4 & 89.1 & 61.1 & 59.3 & 65.4 \\
\hline 38 & 44.4 & 47.4 & 50.0 & 55.6 & 37.5 & 71.4 & 67.9 & 65.7 & 65.0 & 42.9 & 98.2 & 63.9 & 70.4 & 61.5 \\
\hline Pyrimethanil & 75.0 & 36.4 & 21.1 & 90.9 & 100 & 87.5 & 93.9 & 28.0 & 94.4 & 96.2 & 95.7 & 22.2 & 23.8 & 25.0 \\
\hline
\end{tabular}

a A.S: Alternaria solani; F.G: Fusarium graminearum; P.I: Phytophthora infestans; S.S: Sclerotinia sclerotiorum; B.C: Botrytis cinereal; R.S: Rhizoctonia solani; F.O: Fusarium oxysporum f.sp.cucumerinum; C.A: Cercospora arachidicola; P.P: Physalospora piricola; R.C: Rhizoctonia cerealis; B.M: Bipolaris maydis; W.A: Watermelon-anthracnose; F.M: Fusarium moniliforme; P.C: Phytophthora capsica.

Thirdly, when the 4,6-positions of the pyrimidine ring were substituted by a chlorine atom and trifluoroethoxy group (such as compound 17), the antifungal activity was weakened instead of enhanced. Therefore, we further fixed the 4-position of the pyrimidine ring with a chlorine atom or trifluoroethoxy group, and a series of compounds with nitrogen-substituted group at 6-position of the pyrimidine ring were synthesized, such as compounds $\mathbf{1 8}$ (4-Cl, 6-morpholine), 19 (4-Cl, 6- $N^{\prime}, N^{\prime}$-diethyl), 21 (4- $\mathrm{OCH}_{2} \mathrm{CF}_{3}, 6$-pyrrolidinyl) and $22\left(4-\mathrm{OCH}_{2} \mathrm{CF}_{3}, 6-N^{\prime}\right.$-hexyl) (Table 2). Fortunately, compound 19 exhibited broad-spectrum fungicidal activity ( $>60 \%$ inhibitory activities against 13 phytopathogenic fungi), which was better than the inhibitory activities of commercial pyrimethanil ( $>50 \%$ against eight phytopathogenic fungi, Table 2 ).

As another part of our strategy, we found that the antifungal activity of $\mathbf{1 0}(2,6-\mathrm{Cl}$ on the benzoyl ring) is superior to compounds $9(2,4-\mathrm{Cl}$ on the benzoyl ring, Table 2). Compound 25 exhibited broad-spectrum fungicidal activity ( $>50 \%$ inhibitory activities against 13 phytopathogenic fungi). Especially notable was the inhibition activity of compound $\mathbf{2 5}$ against Rhizoctonia cerealis, Phytophthora capsica, Botrytis cinereal and Physalospora piricola. reached 94.9\%, 90.7\%, 89.7\% and 84.2\% 
at $50 \mu \mathrm{g} \mathrm{mL}^{-1}$ respectively, which was comparable to commercial pyrimethanil (Table 2). Unlike the low activity of compound $\mathbf{1 7}(2-\mathrm{Cl})$, the broad-spectrum antifungal activity of compound 25 indicated that 2,6-dichloro substitution on the benzoyl ring was very important for the antifungal activity. However, when we replaced the ethyl group on the nitrogen with benzyl group (compound 29 compared with compound 27) or the 5-position of the pyrimidine ring was substituted with 2-butyl group (compound 30), the antifungal activity decreased significantly (Table 2). This result indicated that groups with large steric hindrance were not conducive to antifungal activity.

In the investigation of the urea bridge unit, a benzoylthiourea derivative (compound 31) and a benzoylguanidine derivative (compound 32) displayed lower antifungal activities than compound 2 and compound 27, respectively (Table 2). Although the phenylurea compound 38 also showed better antifungal activity ( $>50 \%$ inhibitory activities against 10 phytopathogenic fungi), its antifungal activity was lower and its antifungal spectrum smaller than those of compound 19. Finally, because of the broad spectrum and powerful antifungal activity of compounds 19 and 25, the values of $\mathrm{EC}_{50}$ for compounds 19 and 25 against Sclerotinia sclerotiorum, Botrytis cinereal, Physalospora piricola, Rhizoctonia cerealis were determined and are listed in Table 3.

Table 3. Inhibitory Effects of Compounds 19 and 25 against Phytopathogenic Fungi.

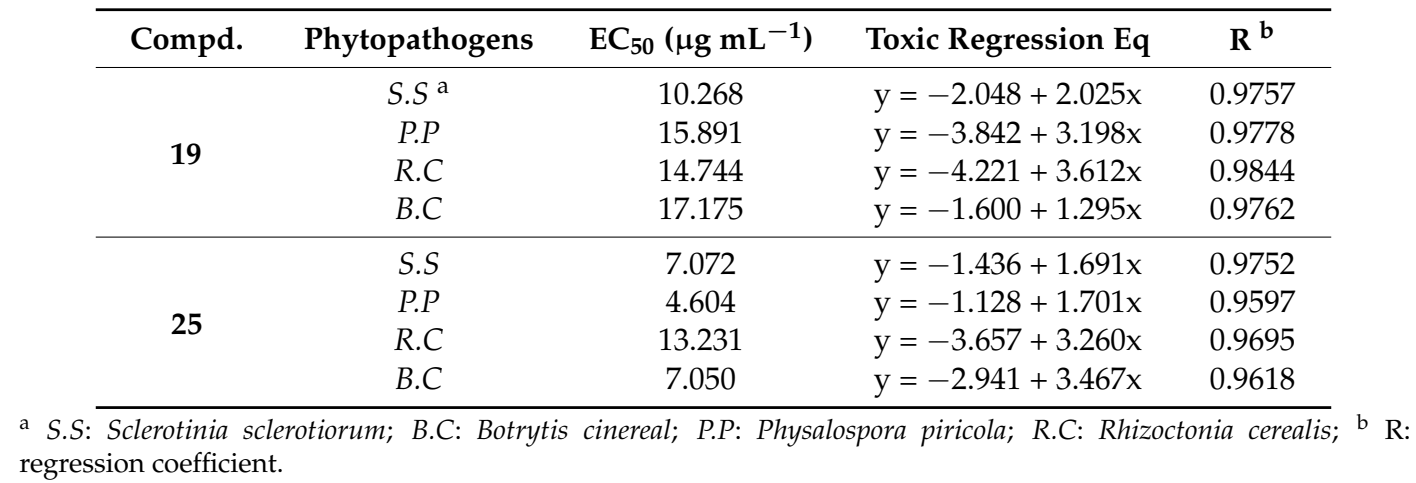

\subsubsection{In Vivo Antifungal Bioassay against Sclerotinia sclerotiorum}

The protective effets of compounds 19 and 25 and carbendazim (positive control) against S. sclerotiorum on detached Brassica oleracea L. leaves were evaluated. The results showed that both compounds 19 and 25 exhibited protective activity on leaves of Brassica oleracea L. (Table 4).

Table 4. In vivo Protective Effects of Compounds 19 and 25 against Sclerotinia sclerotiorum.

\begin{tabular}{ccc}
\hline Compd. & Concn $\left(\mu \mathbf{g ~ m L}^{-\mathbf{1}}\right)$ & Protection Effect $(\mathbf{\%})$ \\
\hline \multirow{2}{*}{$\mathbf{1 9}$} & 3000 & 55.7 \\
& 1500 & 35.2 \\
& 500 & 34.1 \\
\multirow{2}{*}{$\mathbf{2 5}$} & 3000 & 83.0 \\
& 1500 & 35.2 \\
Carbendazim & 500 & 20.5 \\
\hline
\end{tabular}

This protective effect was enhanced with the increasing concentration of the compounds (experimental photos shown in Figure S102 in the Supporting Information). For example, the values of the protective effect for compound 19 were $55.7 \%$ at $3 \mathrm{mg} \mathrm{mL}^{-1}, 35.2 \%$ at $1.5 \mathrm{mg} \mathrm{mL}^{-1}, 34.1 \%$ at $0.5 \mathrm{mg} \mathrm{mL}^{-1}$ and the protective effect values for compound 25 were $83.0 \%$ at $3 \mathrm{mg} \mathrm{mL}^{-1}, 35.2 \%$ at $1.5 \mathrm{mg} \mathrm{mL}^{-1}, 20.5 \%$ at $0.5 \mathrm{mg} \mathrm{mL}^{-1}$ (Table 4). Interestingly, although compound 19 has better in vitro antifungal activity than compound $\mathbf{2 5}$, it appears that compound 25 has a better protective effect. 


\subsubsection{Zebrafish Embryo Toxicity Assay}

The fish embryo acute toxicity test (FET) with zebrafish embryos, which is a general model in ecotoxicology and toxicology had been established in our labs $[15,16]$. The mortality in the positive control was $40 \%$ greater than $30 \%$ and survival in the negative control was $100 \%$. As shown in Table 5 , compound 7 has low toxicity to zebrafish embryos $\left(\mathrm{LC}_{50}=378.387 \mu \mathrm{g} \mathrm{mL} \mathrm{L}^{-1}\right)$, which was what we like to see because of its excellent larvicidal activity to mosquito. At the same time, compound 25 which exhibited broad-spectrum antifungal activity also possesses low toxicity to zebrafish embryos $\left(\mathrm{LC}_{50}=21.668 \mu \mathrm{g} \mathrm{mL}^{-1}\right)$. Fortunately, both compounds 7 and 25 did not affect zebrafish embryo hatching, induce the pericardial cysts and produce abnormality (such as malformation and coagulation) during the zebrafish embryo acute toxicity test.

Table 5. The Zebrafish Embryo Acute Toxicity of Compounds 7 and 25.

\begin{tabular}{cccccc}
\hline Compd. & Time (h) & LC $_{\mathbf{5 0}}\left(\boldsymbol{\mu} \mathbf{g ~ m L}^{-\mathbf{1}}\right)$ & Toxic Regression Eq & $\mathbf{R}$ & 95\% Confidence Limits \\
\hline $\mathbf{7}$ & 96 & 378.387 & $\mathrm{y}=-4.378+1.698 \mathrm{x}$ & 0.9617 & $325.159-369.971$ \\
& 24 & 38.187 & $\mathrm{y}=-2.193+1.387 \mathrm{x}$ & 0.8718 & $23.899-67.569$ \\
\multirow{2}{*}{5} & 48 & 30.435 & $\mathrm{y}=-2.142+1.444 \mathrm{x}$ & 0.9154 & $18.065-48.014$ \\
& 72 & 26.886 & $\mathrm{y}=-2.274+1.591 \mathrm{x}$ & 0.9508 & $16.271-39.781$ \\
& 96 & 21.668 & $\mathrm{y}=-2.131+1.595 \mathrm{x}$ & 0.9762 & $11.777-31.651$ \\
\hline
\end{tabular}

\section{Materials and Methods}

\subsection{Instruments}

${ }^{1} \mathrm{H}-\mathrm{NMR}$ and ${ }^{13} \mathrm{C}-\mathrm{NMR}$ spectra were obtained at 500/126 MHz using a Bruker Avance III 500 spectrometer (Bruker Daltonics, Bremen, Germany) in $\mathrm{CDCl}_{3}$ or DMSO- $d_{6}$ solution with tetramethylsilane as the internal standard. Chemical shift values $(\delta)$ are given in ppm. HRMS data were obtained on FTICR-MS (Ionspec 7.0 T, Lebrilla League, Davis, CA, USA) high resolution mass spectrometer and LQC Advantage MAX multi-stage ion mass spectrometer (Agilent Technologies Inc., CA, USA). The melting points were determined on an X-4 binocular microscope melting point apparatus (Beijing Tech Instruments Co., Beijing, China) and are uncorrected. Yields were not optimized.

\subsection{General Synthesis}

All anhydrous solvents were dried and purified by standard techniques. Chemicals (analytical grade) were purchased from Aladdin (Shanghai, China).

\subsection{General Synthesis Procedure for Substituted 2-Aminopyrimidines Ia-c}

Intermediates Ia-c were prepared according to the literature [17]. A solution of $\mathrm{NaH}(0.35 \mathrm{~g}$, $14.6 \mathrm{mmol})$ in dry THF $(12 \mathrm{~mL})$ was cooled to $0{ }^{\circ} \mathrm{C}$ under $\mathrm{N}_{2}$, then a substituted alcohol (15.2 mmol) was added dropwise. The mixture was stirred for $15 \mathrm{~min}$ while maintaining the temperature at $0{ }^{\circ} \mathrm{C}$. Next, 2-amino-4,6-dichloropyrimidine $(1.0 \mathrm{~g}, 6.1 \mathrm{mmol})$ was added to the solution. The reaction was continued at $62{ }^{\circ} \mathrm{C}$ for $15 \mathrm{~h}$. Then the mixture was cooled to ambient temperature, and quenched with $1 \mathrm{~mL}$ of $1 \mathrm{M}$ hydrochloric acid solution. The mixture was diluted with EtOAc $(20 \mathrm{~mL})$, washed twice with a saturated $\mathrm{NaHCO}_{3}$ solution $(20 \mathrm{~mL})$ and brine $(20 \mathrm{~mL})$, dried with anhydrous $\mathrm{Na}_{2} \mathrm{SO}_{4}$ and evaporated in vacuo. Finally, the residue was purified by silica gel column chromatography (EtOAc/petroleum ether) to afford compounds Ia-c.

4,6-Bis(2,2,2-trifluoroethoxy)pyrimidin-2-amine (Ia). Yellow oil, yield $=94 \% .{ }^{1} \mathrm{H}-\mathrm{NMR}\left(\mathrm{CDCl}_{3}\right) \delta 5.58$ (s, $1 \mathrm{H}, \mathrm{ArH}), 4.96\left(\mathrm{~s}, 2 \mathrm{H}, \mathrm{NH}_{2}\right), 4.57\left(\mathrm{q}, J=8.6 \mathrm{~Hz}, 4 \mathrm{H}, \mathrm{OCH}_{2} \mathrm{CF}_{3}\right)$.

4,6-Dimethoxypyrimidin-2-amine (Ib). White solid, mp 87-89 ${ }^{\circ} \mathrm{C}$, yield $=91 \% .{ }^{1} \mathrm{H}-\mathrm{NMR}\left(\mathrm{CDCl}_{3}\right) \delta 5.46$ (s, $1 \mathrm{H}, \mathrm{ArH}), 5.09\left(\mathrm{~s}, 2 \mathrm{H}, \mathrm{NH}_{2}\right), 3.84\left(\mathrm{~s}, 6 \mathrm{H}, \mathrm{OCH}_{3}\right)$. 
4,6-Diethoxypyrimidin-2-amine (Ic). White solid, $\mathrm{mp} 174-176{ }^{\circ} \mathrm{C}$, yield $=94 \% .{ }^{1} \mathrm{H}-\mathrm{NMR}\left(\mathrm{CDCl}_{3}\right) \delta 5.42$ $(\mathrm{s}, 1 \mathrm{H}, \mathrm{ArH}), 4.95\left(\mathrm{~s}, 2 \mathrm{H}, \mathrm{NH}_{2}\right), 4.23\left(\mathrm{q}, J=7.1 \mathrm{~Hz}, 4 \mathrm{H}, \mathrm{OCH}_{2} \mathrm{CH}_{3}\right), 1.35\left(\mathrm{t}, J=7.1 \mathrm{~Hz}, 6 \mathrm{H}, \mathrm{OCH}_{2} \mathrm{CH}_{3}\right)$.

\subsection{General Synthesis Procedure for Substituted 2-Aminopyrimidines Id-f}

Intermediates Id-f fere prepared by a method similar to that for intermediates Ia-c. A solution of $\mathrm{NaH}(5.8 \mathrm{mmol})$ in dry THF $(12 \mathrm{~mL})$ was cooled to $0{ }^{\circ} \mathrm{C}$ under $\mathrm{N}_{2}$, then a substituted alcohol $(6.1 \mathrm{mmol})$ was added dropwise. The mixture was stirred for $15 \mathrm{~min}$ while maintaining the temperature at $0{ }^{\circ} \mathrm{C}$. Next, 2-amino-4-chloro-6- substituted-pyrimidine $(5.8 \mathrm{mmol})$ was added to the solution. The reaction was continued at $62{ }^{\circ} \mathrm{C}$ for $15 \mathrm{~h}$. Then the mixture was cooled to ambient temperature, and quenched with $1 \mathrm{~mL}$ of $1 \mathrm{M}$ hydrochloric acid solution. The mixture was diluted with EtOAc $(20 \mathrm{~mL})$, washed twice with a saturated $\mathrm{NaHCO}_{3}$ solution $(20 \mathrm{~mL})$ and brine $(20 \mathrm{~mL})$, dried with anhydrous $\mathrm{Na}_{2} \mathrm{SO}_{4}$ and evaporated in vacuo. Finally, the residue was purified by silica gel column chromatography (EtOAc/petroleum ether) to afford compounds Id-f.

4-Chloro-6-methoxypyrimidin-2-amine (Id). White solid, mp $165-167{ }^{\circ} \mathrm{C}$, yield $=84 \% .{ }^{1} \mathrm{H}-\mathrm{NMR}\left(\mathrm{CDCl}_{3}\right)$ $\delta 6.04(\mathrm{~s}, 1 \mathrm{H}, \mathrm{ArH}), 5.35-5.14\left(\mathrm{~m}, 2 \mathrm{H}, \mathrm{NH}_{2}\right), 3.81\left(\mathrm{~s}, 3 \mathrm{H}, \mathrm{OCH}_{3}\right)$.

4-Methyl-6-(2,2,2-trifluoroethoxy)pyrimidin-2-amine (Ie). White solid, mp $109-111{ }^{\circ} \mathrm{C}$, yield $=69 \%$. ${ }^{1} \mathrm{H}-\mathrm{NMR}\left(\mathrm{CDCl}_{3}\right) \delta 6.42(\mathrm{~s}, 1 \mathrm{H}, \mathrm{ArH}), 5.50\left(\mathrm{~s}, 2 \mathrm{H}, \mathrm{NH}_{2}\right), 4.60\left(\mathrm{q}, J=8.4 \mathrm{~Hz}, 2 \mathrm{H}, \mathrm{OCH}_{2} \mathrm{CF}_{3}\right), 2.18$ $\left(\mathrm{s}, 3 \mathrm{H}, \mathrm{CH}_{3}\right)$.

4-Chloro-6-(2,2,2-trifluoroethoxy)pyrimidin-2-amine (If). White solid, $\mathrm{mp} 82-83{ }^{\circ} \mathrm{C}$, yield $=76 \% .{ }^{1} \mathrm{H}-\mathrm{NMR}$ $\left(\mathrm{CDCl}_{3}\right) \delta 6.23(\mathrm{~s}, 1 \mathrm{H}, \mathrm{ArH}), 5.59\left(\mathrm{~s}, 2 \mathrm{H}, \mathrm{NH}_{2}\right), 4.70\left(\mathrm{q}, J=8.4 \mathrm{~Hz}, 2 \mathrm{H}, \mathrm{OCH}_{2} \mathrm{CF}_{3}\right)$.

\subsection{General Synthesis Procedure for Substituted 2-Aminopyrimidines Ig-m}

Intermediates Ig- $\mathbf{m}$ were prepared according to [18]. A suspension of $\mathrm{CuI}(5.8 \mathrm{mg}, 0.03$ $\mathrm{mmol}), \mathrm{K}_{2} \mathrm{CO}_{3}(0.41 \mathrm{~g}, 2.9 \mathrm{mmol})$, the corresponding amine $(0.21 \mathrm{~g}, 2.4 \mathrm{mmol})$ and substituted 2-aminopyrimidines $(0.4 \mathrm{~g}, 2.4 \mathrm{mmol})$ in $2 \mathrm{~mL} \mathrm{DMF}$ was stirred for $30 \mathrm{~min}$ at room temperature and then the mixture was heated to $110{ }^{\circ} \mathrm{C}$. At the end of the reaction, as monitored by TLC, the reaction mixture was cooled to room temperature and diluted with $20 \mathrm{~mL}$ of EtOAc, washed twice with a saturated $\mathrm{NaHCO}_{3}$ solution $(20 \mathrm{~mL})$ and brine $(20 \mathrm{~mL})$, dried with anhydrous $\mathrm{MgSO}_{4}$ and evaporated in vacuo. Finally, the residue was purified by silica gel column chromatography to afford compound Ig-m.

4-Chloro-6-morpholinopyrimidin-2-amine (Ig). White solid, mp $210-212{ }^{\circ} \mathrm{C}$, yield $=79 \%$. ${ }^{1} \mathrm{H}-\mathrm{NMR}$ $\left(\mathrm{CDCl}_{3}\right) \delta 5.94(\mathrm{~s}, 1 \mathrm{H}, \mathrm{ArH}), 5.00\left(\mathrm{~s}, 2 \mathrm{H}, \mathrm{NH}_{2}\right), 3.79-3.68(\mathrm{~m}, 4 \mathrm{H}$, morpholine), $3.55(\mathrm{t}, J=4.9 \mathrm{~Hz}, 4 \mathrm{H}$, morpholine).

6-Chloro- $\mathrm{N}^{4}, \mathrm{~N}^{4}$-diethylpyrimidine-2,4-diamine (Ih). White solid, $\mathrm{mp} 104-106{ }^{\circ} \mathrm{C}$, yield $=81 \% .{ }^{1} \mathrm{H}-\mathrm{NMR}$ $\left(\mathrm{CDCl}_{3}\right) \delta 5.84(\mathrm{~s}, 1 \mathrm{H}, \mathrm{ArH}), 5.04\left(\mathrm{~s}, 2 \mathrm{H}, \mathrm{NH}_{2}\right), 3.42\left(\mathrm{~s}, 4 \mathrm{H}, \mathrm{CH}_{2} \mathrm{CH}_{3}\right), 1.15\left(\mathrm{t}, J=7.1 \mathrm{~Hz}, 6 \mathrm{H}, \mathrm{CH}_{2} \mathrm{CH}_{3}\right)$.

$\mathrm{N}^{4}$-(Pyrrolidin-1-yl)-6-(2,2,2-trifluoroethoxy)pyrimidine-2,4-diamine (Ii). White solid, mp 118-120 ${ }^{\circ} \mathrm{C}$, yield $=51 \% .{ }^{1} \mathrm{H}-\mathrm{NMR}\left(\mathrm{CDCl}_{3}\right) \delta 5.21(\mathrm{~s}, 1 \mathrm{H}, \mathrm{ArH}), 4.82\left(\mathrm{~s}, 2 \mathrm{H}, \mathrm{NH}_{2}\right), 4.67\left(\mathrm{q}, J=8.7 \mathrm{~Hz}, 2 \mathrm{H}, \mathrm{OCH}_{2} \mathrm{CF}_{3}\right)$, $3.38\left(\mathrm{~s}, 4 \mathrm{H}\right.$, tetrahydropyrrole), $1.93\left(\mathrm{~d}, J=5.9 \mathrm{~Hz}, 4 \mathrm{H}\right.$, tetrahydropyrrole). ${ }^{13} \mathrm{C}-\mathrm{NMR}\left(\mathrm{CDCl}_{3}\right) \delta 168.7$, 163.3, 162.0, 124.9, 122.7, 62.0, 61.7, 61.4, 61.1, 46.5, 25.3. HRMS (ESI): $m / z$ calcd for $\mathrm{C}_{10} \mathrm{H}_{13} \mathrm{~F}_{3} \mathrm{~N}_{4} \mathrm{O}$ $[\mathrm{M}+\mathrm{H}]^{+}:$263.1120, found 263.1136.

$\mathrm{N}^{4}$-Hexyl-6-(2,2,2-trifluoroethoxy)pyrimidine-2,4-diamine (Ij). Yellow oil, yield $=53 \% .{ }^{1} \mathrm{H}-\mathrm{NMR}\left(\mathrm{CDCl}_{3}\right)$ $\delta 5.24(\mathrm{~s}, 1 \mathrm{H}, \mathrm{ArH}), 5.13(\mathrm{~s}, 1 \mathrm{H}, \mathrm{NH}), 5.06\left(\mathrm{~s}, 2 \mathrm{H}, \mathrm{NH}_{2}\right), 4.65\left(\mathrm{q}, J=8.6 \mathrm{~Hz}, 2 \mathrm{H}, \mathrm{OCH}_{2} \mathrm{CF}_{3}\right), 3.12$ $\left(\mathrm{q}, J=6.7 \mathrm{~Hz}, 2 \mathrm{H}, \mathrm{N}-\mathrm{CH}_{2}\left(\mathrm{CH}_{2}\right)_{4} \mathrm{CH}_{3}\right), 1.77-1.42\left(\mathrm{~m}, 2 \mathrm{H}, \mathrm{N}-\left(\mathrm{CH}_{2}\right)_{4} \mathrm{CH}_{2} \mathrm{CH}_{3}\right), 1.44-1.11(\mathrm{~m}, 6 \mathrm{H}$, $\left.\mathrm{N}-\mathrm{CH}_{2}\left(\mathrm{CH}_{2}\right)_{3} \mathrm{CH}_{2} \mathrm{CH}_{3}\right), 0.88\left(\mathrm{t}, J=6.8 \mathrm{~Hz}, 3 \mathrm{H}, \mathrm{N}-\left(\mathrm{CH}_{2}\right)_{5} \mathrm{CH}_{3}\right) .{ }^{13} \mathrm{C}-\mathrm{NMR}\left(\mathrm{CDCl}_{3}\right) \delta$ 169.4, 165.6, 162.1, 124.7, 122.5, 62.0, 61.7, 61.5, 61.2, 41.8, 31.5, 29.2, 26.6, 22.6, 14.0. HRMS (ESI): $\mathrm{m} / \mathrm{z}$ calcd for $\mathrm{C}_{10} \mathrm{H}_{19} \mathrm{~F}_{3} \mathrm{~N}_{4} \mathrm{O}[\mathrm{M}+\mathrm{H}]^{+}:$293.1589, found 293.1593. 
$\mathrm{N}^{4}, \mathrm{~N}^{4}$-Diethyl-6-(2,2,2-trifluoroethoxy)pyrimidine-2,4-diamine (Ik). Yellow oil, yield $=49 \%$. ${ }^{1} \mathrm{H}$-NMR $\left(\mathrm{CDCl}_{3}\right) \delta 5.32(\mathrm{~s}, 1 \mathrm{H}, \mathrm{ArH}), 4.78\left(\mathrm{~s}, 2 \mathrm{H}, \mathrm{NH}_{2}\right), 4.72-4.61\left(\mathrm{~m}, 2 \mathrm{H}, \mathrm{OCH}_{2} \mathrm{CF}_{3}\right), 3.41(\mathrm{q}, J=7.0 \mathrm{~Hz}, 4 \mathrm{H}$, $\left.\mathrm{CH}_{2} \mathrm{CH}_{3}\right), 1.13\left(\mathrm{t}, J=7.1 \mathrm{~Hz}, 6 \mathrm{H}, \mathrm{CH}_{2} \mathrm{CH}_{3}\right) .{ }^{13} \mathrm{C}-\mathrm{NMR}\left(\mathrm{CDCl}_{3}\right) \delta 170.4,169.3,163.9,124.9,81.7,62.3$, 62.0, 61.7, 61.4, 42.1, 12.9. HRMS (ESI): $m / z$ calcd for $\mathrm{C}_{10} \mathrm{H}_{15} \mathrm{~F}_{3} \mathrm{~N}_{4} \mathrm{O}[\mathrm{M}+\mathrm{H}]^{+}: 265.1276$, found 265.1280.

$\mathrm{N}^{4}$-Benzyl-6-(2,2,2-trifluoroethoxy)pyrimidine-2,4-diamine (Il). Yellow oil, yield $=55 \% .{ }^{1} \mathrm{H}-\mathrm{NMR}\left(\mathrm{CDCl}_{3}\right)$ $\delta$ 7.36-7.15 (m, 5H, Ph), $5.66(\mathrm{~s}, 1 \mathrm{H}, \mathrm{ArH}), 5.22(\mathrm{~s}, 1 \mathrm{H}, \mathrm{NH}), 5.02\left(\mathrm{~s}, 2 \mathrm{H}, \mathrm{NH}_{2}\right), 4.59(\mathrm{q}, J=8.6 \mathrm{~Hz}, 2 \mathrm{H}$, $\left.\mathrm{OCH}_{2} \mathrm{CF}_{3}\right), 4.31\left(\mathrm{~d}, J=6.0 \mathrm{~Hz}, 2 \mathrm{H}, \mathrm{PhCH}_{2}\right) .{ }^{13} \mathrm{C}-\mathrm{NMR}\left(\mathrm{CDCl}_{3}\right) \delta 169.4,165.6,162.2,138.2,128.7,127.5$, 127.1, 126.9, 124.7, 122.5, 120.3, 62.0, 61.7, 61.4, 61.2, 45.6. HRMS (ESI): $m / z$ calcd for $\mathrm{C}_{13} \mathrm{H}_{13} \mathrm{~F}_{3} \mathrm{~N}_{4} \mathrm{O}$ $[\mathrm{M}+\mathrm{H}]^{+}:$299.1120, found 299.1119.

$N^{4}$-Benzyl-N $N^{4}$-ethyl-6-(2,2,2-trifluoroethoxy)pyrimidine-2,4-diamine $(\mathbf{I m})$. Yellow oil, yield $=43 \% .{ }^{1} \mathrm{H}-\mathrm{NMR}$ $\left(\mathrm{DMSO}-d_{6}\right) \delta 7.65-7.03(\mathrm{~m}, 5 \mathrm{H}, \mathrm{Ph}), 6.23\left(\mathrm{~s}, 2 \mathrm{H}, \mathrm{NH}_{2}\right), 5.31(\mathrm{~s}, 1 \mathrm{H}, \mathrm{ArH}), 4.86(\mathrm{q}, J=9.2 \mathrm{~Hz}, 2 \mathrm{H}$, $\left.\mathrm{OCH}_{2} \mathrm{CF}_{3}\right), 4.69\left(\mathrm{~s}, 2 \mathrm{H}, \mathrm{PhCH}_{2}\right), 3.41\left(\mathrm{~s}, 2 \mathrm{H}, \mathrm{CH}_{2} \mathrm{CH}_{3}\right), 1.04\left(\mathrm{t}, J=7.0 \mathrm{~Hz}, 3 \mathrm{H}, \mathrm{CH}_{2} \mathrm{CH}_{3}\right) .{ }^{13} \mathrm{C}-\mathrm{NMR}$ (DMSO- $d_{6}$ ) $\delta 169.6,165.1,163.1,129.4,127.9,127.7,126.1,123.9,75.6,61.3,61.1,42.7,13.4$. HRMS (ESI): $m / z$ calcd for $\mathrm{C}_{15} \mathrm{H}_{17} \mathrm{~F}_{3} \mathrm{~N}_{4} \mathrm{O}[\mathrm{M}+\mathrm{H}]^{+}: 327.1433$, found 327.1429.

\subsection{Synthesis of 5-(sec-Butyl)-4,6-dichloropyrimidin-2-amine (Intermediate In)}

Intermediate In was prepared according to [19]. Elemental sodium (0.30 g, $13.0 \mathrm{mmol})$ was added into absolute ethanol $(7 \mathrm{~mL})$ under $\mathrm{N}_{2}$ while being intensively stirred with a magnetic stirrer. After all the sodium was dissolved and the reaction mixture was cooled to room temperature, guanidine hydrochloride $(0.49 \mathrm{~g}, 5.1 \mathrm{mmol})$ was added under intensive stirring, followed by the diethyl sec-butylmalonate $(1.9 \mathrm{~g}, 4.6 \mathrm{mmol})$. The reaction mixture was further stirred intensively at room temperature. After another $4 \mathrm{~h}$, absolute ethanol $(5 \mathrm{~mL})$ was added and the reaction mixture was refluxed for $1 \mathrm{~h}$. Afterward, ethanol was evaporated on a vacuum rotary evaporator and water $(12 \mathrm{~mL})$ was added to the reaction mixture. After stirring, the product was dissolved. The obtained mixture was subsequently neutralized by acetic acid and then this mixture was heated under reflux for $10 \mathrm{~min}$ and then cooled to room temperature. This heating and cooling was repeated twice to get a well-filterable solid. The solid was filtered off, washed with water $(2 \times 50 \mathrm{~mL})$, ethanol $(2 \times 50 \mathrm{~mL})$, and acetone $(2 \times 50 \mathrm{~mL})$. The product was dried under high vacuum for 2 days. Subsequently, the aboveobtained solid (4.6 mmol) was suspended under $\mathrm{N}_{2}$ in a solution of (chloro-methylene)dimethyl ammonium chloride $(4.8 \mathrm{~g}, 37.1 \mathrm{mmol})$ in chloroform $(19 \mathrm{~mL})$. The reaction mixture was subsequently heated at reflux for $4 \mathrm{~h}$, during which the starting material was completely dissolved. The reaction mixture was cooled to the room temperature, poured into ice and rapidly neutralized with a saturated aqueous $\mathrm{NaHCO}_{3}$ solution. The obtained mixture was quickly transferred into a separatory funnel and immediately extracted with chloroform $(3 \times 20 \mathrm{~mL})$. The organic layers were combined together, dried over $\mathrm{MgSO}_{4}$, filtered and concentrated down on a rotary evaporator. This crude residue was dissolved in the mixture of ethanol $(9 \mathrm{~mL})$ and $37 \%$ aqueous $\mathrm{HCl}(0.9 \mathrm{~mL})$. The reaction mixture was heated at $50{ }^{\circ} \mathrm{C}$ for $2 \mathrm{~h}$. After that, water $(14 \mathrm{~mL})$ was added and the reaction mixture was stirred for $10 \mathrm{~min}$. The precipitated product was filtered off and washed with a water/ethanol mixture $(v / v=1 / 1$, $2 \times 5 \mathrm{~mL}), 5 \%$ aqueous solution of $\mathrm{NaHCO}_{3}(5 \mathrm{~mL})$. The product was subsequently recrystallized from aqueous ethanol, filtered off, washed with a water/ethanol mixture $(v / v=1 / 1,5 \mathrm{~mL})$ and the solid recrystallized from ethanol to give intermediate $\mathrm{In}$ as a white solid, $\mathrm{mp} 157-159{ }^{\circ} \mathrm{C}$, yield $=73 \%$. ${ }^{1} \mathrm{H}-\mathrm{NMR}\left(\mathrm{CDCl}_{3}\right) \delta 5.98\left(\mathrm{~d}, J=6.5 \mathrm{~Hz}, 2 \mathrm{H}, \mathrm{NH}_{2}\right), 3.27(\mathrm{dq}, J=6.9,3.7,2.9 \mathrm{~Hz}, 1 \mathrm{H}, \mathrm{CH}), 1.95-1.75(\mathrm{~m}$, $\left.1 \mathrm{H}, \mathrm{CHCH}_{2}\right), 1.63\left(\mathrm{dddd}, J=16.0,9.0,4.7,2.3 \mathrm{~Hz}, 1 \mathrm{H}, \mathrm{CHCH}_{2}\right), 1.24\left(\mathrm{dt}, J=7.4,2.3 \mathrm{~Hz}, 3 \mathrm{H}, \mathrm{CHCH}_{3}\right)$, 0.77 (ddd, $J=9.1,5.7,2.1 \mathrm{~Hz}, 3 \mathrm{H}, \mathrm{CHCH}_{2} \mathrm{CH}_{3}$ ).

\subsection{Synthesis of 5-(sec-Butyl)-6-chloro- $\mathrm{N}^{4}, N^{4}$-diethylpyrimidine-2,4-diamine (Intermediate Io)}

Intermediate Io was prepared by a method similar to that used for compounds Ig-m. Yellow oil, yield $=42 \% .{ }^{1} \mathrm{H}-\mathrm{NMR}\left(\right.$ DMSO- $\left.d_{6}\right) \delta 6.44(\mathrm{~s}, 2 \mathrm{H}), 3.23(\mathrm{dd}, J=13.7,6.9 \mathrm{~Hz}, 2 \mathrm{H}), 3.05(\mathrm{dd}, J=13.7,7.0 \mathrm{~Hz}$, 2H), $2.79(\mathrm{q}, J=7.4 \mathrm{~Hz}, 1 \mathrm{H}), 1.79-1.61(\mathrm{~m}, 2 \mathrm{H}), 1.31(\mathrm{~d}, J=7.1 \mathrm{~Hz}, 3 \mathrm{H}), 1.05(\mathrm{t}, J=7.0 \mathrm{~Hz}, 6 \mathrm{H}), 0.71(\mathrm{t}$, 
$J=7.4 \mathrm{~Hz}, 3 \mathrm{H}$ ). ${ }^{13} \mathrm{C}-\mathrm{NMR}\left(\mathrm{DMSO}-d_{6}\right) \delta 170.8,160.8,159.7,114.1,45.8,34.4,28.3,19.0,14.0,13.6$. HRMS (ESI): $m / z$ calcd for $\mathrm{C}_{12} \mathrm{H}_{21} \mathrm{ClN}_{4}[\mathrm{M}+\mathrm{Na}]^{+}: 279.1352$, found 279.1356 .

\subsection{Synthesis of 5-(sec-Butyl)-4-chloro-6-(2,2,2-trifluoroethoxy)pyrimidin-2-amine (Intermediate Ip)}

Intermediate Ip was prepared by a method similar to that used for compounds Id-f. White solid, mp 53-54 ${ }^{\circ} \mathrm{C}$, yield $=40 \% .{ }^{1} \mathrm{H}-\mathrm{NMR}\left(\mathrm{DMSO}_{6} \mathrm{~d}_{6}\right) \delta 7.00(\mathrm{~s}, 2 \mathrm{H}, \mathrm{NH} 2), 4.98\left(\mathrm{q}, J=9.0 \mathrm{~Hz}, 2 \mathrm{H}, \mathrm{OCH}_{2} \mathrm{CF}_{3}\right)$, $3.02(\mathrm{dt}, J=9.3,6.7 \mathrm{~Hz}, 1 \mathrm{H}, \mathrm{CH}), 1.76-1.62\left(\mathrm{~m}, 1 \mathrm{H}, \mathrm{CHCH}_{2}\right), 1.56\left(\mathrm{ddd}, J=13.6,7.6,6.4 \mathrm{~Hz}, 1 \mathrm{H}, \mathrm{CHCH}_{2}\right)$, $1.18\left(\mathrm{~d}, J=7.1 \mathrm{~Hz}, 3 \mathrm{H}, \mathrm{CHCH}_{3}\right), 0.75\left(\mathrm{t}, J=7.4 \mathrm{~Hz}, 3 \mathrm{H}, \mathrm{CHCH}_{2} \mathrm{CH}_{3}\right) .{ }^{13} \mathrm{C}-\mathrm{NMR}\left(\mathrm{DMSO}-d_{6}\right) \delta 167.6$, $160.9,160.7,109.8,62.8,62.5,62.2,62.0,36.5,27.9,19.4,13.3$. HRMS (ESI): $m / z$ calcd for $\mathrm{C}_{10} \mathrm{H}_{13} \mathrm{ClF}_{3} \mathrm{~N}_{3} \mathrm{O}$ $[\mathrm{M}+\mathrm{H}]^{+}: 284.0777$, found 284.0799 .

\subsection{General Synthesis Procedure for Target Compounds 1-30}

Compounds 1-30 were synthesized according to [20,21]. Substituted benzamide (5.0 mmol) and 1,2-dichloroethane $(10 \mathrm{~mL})$ were added to a $100 \mathrm{~mL}$ three-necked flask under $\mathrm{N}_{2}$. The reaction mixture was cooled to $0{ }^{\circ} \mathrm{C}$ and oxalyl chloride $(1.27 \mathrm{~g}, 10.0 \mathrm{mmol})$ was added dropwise under stirring, then the mixture was stirred at room temperature for $1 \mathrm{~h}$, left standing at $60^{\circ} \mathrm{C}$ for $3 \mathrm{~h}$ and refluxed for $1 \mathrm{~h}$. The solvent and excess oxalyl chloride were evaporated under reduced pressure to give a yellow transparent liquid. Anhydrous dichloromethane $(5 \mathrm{~mL})$ was added to the residue, then a substituted 2-aminopyrimidine $(2.5 \mathrm{mmol})$ was added to the system and reacted at room temperature for $12 \mathrm{~h}$. The solvent was removed under reduced pressure and the residue was purified by flash column chromatography on silica gel to give compounds 1-30.

$\mathrm{N}$-((4,6-bis(2,2,2-Trifluoroethoxy)pyrimidin-2-yl)carbamoyl)-2,6-difluorobenzamide (1). White solid, $\mathrm{mp}$ 163-164 ${ }^{\circ} \mathrm{C}$, yield $=83 \% .{ }^{1} \mathrm{H}-\mathrm{NMR}\left(\mathrm{DMSO}-d_{6}\right) \delta 11.65(\mathrm{~s}, 1 \mathrm{H}, \mathrm{NH}), 10.77(\mathrm{~s}, 1 \mathrm{H}, \mathrm{NH}), 7.76-7.57(\mathrm{~m}$, $1 \mathrm{H}, \mathrm{Ph}), 7.26(\mathrm{t}, J=8.3 \mathrm{~Hz}, 2 \mathrm{H}, \mathrm{Ph}), 6.40(\mathrm{~s}, 1 \mathrm{H}, \mathrm{ArH}), 5.07\left(\mathrm{q}, J=8.9 \mathrm{~Hz}, 4 \mathrm{H}, \mathrm{OCH}_{2} \mathrm{CF}_{3}\right) .{ }^{13} \mathrm{C}-\mathrm{NMR}$ (DMSO- $d_{6}$ ) $\delta 170.9,160.6,156.3,148.9,134.2,125.6,123.4,113.2,113.0,86.8,63.7,63.4,63.1,62.8$. HRMS (ESI): $m / z$ calcd for $\mathrm{C}_{16} \mathrm{H}_{10} \mathrm{~F}_{8} \mathrm{~N}_{4} \mathrm{O}_{4}[\mathrm{M}+\mathrm{H}]^{+}: 475.0652$, found 475.0641 .

$\mathrm{N}$-((4,6-bis(2,2,2-Trifluoroethoxy)pyrimidin-2-yl)carbamoyl)-2-chlorobenzamide (2). White solid, $\mathrm{mp}$ 173-175 ${ }^{\circ} \mathrm{C}$, yield $=95 \% .{ }^{1} \mathrm{H}-\mathrm{NMR}\left(\mathrm{DMSO}-d_{6}\right) \delta 11.47(\mathrm{~s}, 1 \mathrm{H}, \mathrm{NH}), 10.96(\mathrm{~s}, 1 \mathrm{H}, \mathrm{NH}), 7.65(\mathrm{dd}$, $J=7.6,1.6 \mathrm{~Hz}, 1 \mathrm{H}, \mathrm{Ph}), 7.61-7.52(\mathrm{~m}, 2 \mathrm{H}, \mathrm{Ph}), 7.48(\mathrm{td}, J=7.3,1.7 \mathrm{~Hz}, 1 \mathrm{H}, \mathrm{Ph}), 6.39(\mathrm{~s}, 1 \mathrm{H}, \mathrm{ArH}), 5.07(\mathrm{q}$, $\left.J=8.9 \mathrm{~Hz}, 4 \mathrm{H}, \mathrm{OCH}_{2} \mathrm{CF}_{3}\right) .{ }^{13} \mathrm{C}-\mathrm{NMR}$ (DMSO- $\left.d_{6}\right) \delta 170.9,169.2,156.3,149.0,135.6,133.1,130.7,130.0$, 128.2, 125.7, 123.4, 86.8, 63.6, 63.3, 63.0, 62.8. HRMS (ESI): $m / z$ calcd for $\mathrm{C}_{16} \mathrm{H}_{11} \mathrm{ClF}_{6} \mathrm{~N}_{4} \mathrm{O}_{4}[\mathrm{M}+\mathrm{H}]^{+}$: 473.0451, found 473.0440 .

$\mathrm{N}-((4,6$-bis(2,2,2-Trifluoroethoxy)pyrimidin-2-yl)carbamoyl)-2-bromobenzamide (3). White solid, $\mathrm{mp}$ 110-112 ${ }^{\circ} \mathrm{C}$, yield $=77 \% .{ }^{1} \mathrm{H}-\mathrm{NMR}\left(\mathrm{DMSO}-d_{6}\right) \delta 11.46(\mathrm{~s}, 1 \mathrm{H}, \mathrm{NH}), 10.96(\mathrm{~s}, 1 \mathrm{H}, \mathrm{NH}), 7.73(\mathrm{~d}, J=7.9 \mathrm{~Hz}$, $1 \mathrm{H}, \mathrm{Ph}), 7.62(\mathrm{dd}, J=7.4,1.7 \mathrm{~Hz}, 1 \mathrm{H}, \mathrm{Ph}), 7.57-7.42(\mathrm{~m}, 2 \mathrm{H}, \mathrm{Ph}), 6.39(\mathrm{~s}, 1 \mathrm{H}, \mathrm{ArH}), 5.07$ (q, J = 8.9 Hz, $4 \mathrm{H}, \mathrm{OCH}_{2} \mathrm{CF}_{3}$ ). ${ }^{13} \mathrm{C}-\mathrm{NMR}$ (DMSO- $d_{6}$ ) $\delta 170.8,170.0,156.2,149.0,137.8,133.7,133.0,129.8,128.6,125.6$, 119.5, 86.8, 63.6, 63.3, 63.0, 62.7. HRMS (ESI): $m / z$ calcd for $\mathrm{C}_{16} \mathrm{H}_{11} \mathrm{BrF}_{6} \mathrm{~N}_{4} \mathrm{O}_{4}[\mathrm{M}+\mathrm{Na}]^{+}: 538.9766$, found 538.9769 .

$\mathrm{N}$-((4,6-bis(2,2,2-Trifluoroethoxy)pyrimidin-2-yl)carbamoyl)-2-iodobenzamide (4). White solid, $\mathrm{mp}$ $115-116{ }^{\circ} \mathrm{C}$, yield $=88 \% .{ }^{1} \mathrm{H}-\mathrm{NMR}\left(\mathrm{DMSO}_{6}\right) \delta 11.42(\mathrm{~s}, 1 \mathrm{H}, \mathrm{NH}), 11.01(\mathrm{~s}, 1 \mathrm{H}, \mathrm{NH}), 7.95(\mathrm{dd}$, $J=7.9,1.0 \mathrm{~Hz}, 1 \mathrm{H}, \mathrm{Ph}), 7.72-7.38(\mathrm{~m}, 2 \mathrm{H}, \mathrm{Ph}), 7.28$ (td, $J=7.6,1.9 \mathrm{~Hz}, 1 \mathrm{H}, \mathrm{Ph}), 6.39(\mathrm{~s}, 1 \mathrm{H}, \mathrm{ArH}), 5.07$ $\left(\mathrm{q}, J=8.9 \mathrm{~Hz}, 4 \mathrm{H}, \mathrm{OCH}_{2} \mathrm{CF}_{3}\right) .{ }^{13} \mathrm{C}-\mathrm{NMR}\left(\mathrm{DMSO}-d_{6}\right) \delta 171.7,170.9,156.3,149.1,141.7,140.2,132.9$, 129.2, 129.1, 125.7, 125.4, 93.9, 86.8, 63.6, 63.4, 63.1, 62.8. HRMS (ESI): $m / z$ calcd for $\mathrm{C}_{16} \mathrm{H}_{11} \mathrm{~F}_{6} \mathrm{IN}_{4} \mathrm{O}_{4}$ $[\mathrm{M}+\mathrm{Na}]^{+}: 586.9627$, found 586.9603 .

$\mathrm{N}$-((4,6-bis(2,2,2-Trifluoroethoxy)pyrimidin-2-yl)carbamoyl)-4-chlorobenzamide (5). White solid, $\mathrm{mp}$ 162-163 ${ }^{\circ} \mathrm{C}$, yield $=94 \% .{ }^{1} \mathrm{H}-\mathrm{NMR}\left(\mathrm{DMSO}_{-} \mathrm{d}_{6}\right) \delta 11.42(\mathrm{~s}, 1 \mathrm{H}, \mathrm{NH}), 11.22(\mathrm{~s}, 1 \mathrm{H}, \mathrm{NH}), 8.02(\mathrm{~d}, J=8.5 \mathrm{~Hz}$, $2 \mathrm{H}, \mathrm{Ph}), 7.60(\mathrm{~d}, J=8.6 \mathrm{~Hz}, 2 \mathrm{H}, \mathrm{Ph}), 6.36(\mathrm{~s}, 1 \mathrm{H}, \mathrm{ArH}), 5.07\left(\mathrm{q}, J=8.9 \mathrm{~Hz}, 4 \mathrm{H}, \mathrm{OCH}_{2} \mathrm{CF}_{3}\right) .{ }^{13} \mathrm{C}-\mathrm{NMR}$ 
(DMSO- $d_{6}$ ) $\delta 170.8,168.0,156.4,149.4,139.1,132.1,131.1,129.6,125.6,86.4,63.6,63.3,63.0,62.8$. HRMS (ESI): $m / z$ calcd for $\mathrm{C}_{16} \mathrm{H}_{11} \mathrm{ClF}_{6} \mathrm{~N}_{4} \mathrm{O}_{4}[\mathrm{M}+\mathrm{H}]^{+}: 473.0451$, found 473.0442 .

$\mathrm{N}$-((4,6-bis(2,2,2-Trifluoroethoxy)pyrimidin-2-yl)carbamoyl)-2-(trifluoromethyl)benzamide (6). White solid, mp 51-53 ${ }^{\circ} \mathrm{C}$, yield $=68 \% .{ }^{1} \mathrm{H}-\mathrm{NMR}\left(\mathrm{DMSO}_{6}\right) \delta 11.55(\mathrm{~s}, 1 \mathrm{H}, \mathrm{NH}), 10.91(\mathrm{~s}, 1 \mathrm{H}, \mathrm{NH}), 7.88(\mathrm{~d}$, $J=7.7 \mathrm{~Hz}, 1 \mathrm{H}, \mathrm{Ph}), 7.85-7.71(\mathrm{~m}, 3 \mathrm{H}, \mathrm{Ph}), 6.40(\mathrm{~s}, 1 \mathrm{H}, \mathrm{ArH}), 5.07\left(\mathrm{q}, J=8.9 \mathrm{~Hz}, 4 \mathrm{H}, \mathrm{OCH}_{2} \mathrm{CF}_{3}\right)$. ${ }^{13}$ C-NMR (DMSO- $d_{6}$ ) $\delta 170.8,170.0,156.3,149.0,134.8,133.5,131.9,129.3,127.4,127.1,126.8,126.5$, 126.3, 125.6, 123.4, 86.7, 63.6, 63.3, 63.0, 62.8. HRMS (ESI): $m / z$ calcd for $\mathrm{C}_{17} \mathrm{H}_{11} \mathrm{~F}_{9} \mathrm{~N}_{4} \mathrm{O}_{4}[\mathrm{M}+\mathrm{H}]^{+}$: 507.0715, found 507.0711.

N-((4,6-bis(2,2,2-Trifluoroethoxy)pyrimidin-2-yl)carbamoyl)-4-(trifluoromethyl)benzamide (7). White solid, mp 167-169 ${ }^{\circ} \mathrm{C}$, yield $=93 \% .{ }^{1} \mathrm{H}-\mathrm{NMR}\left(\mathrm{DMSO}_{-} \mathrm{d}_{6}\right) \delta 11.61(\mathrm{~s}, 1 \mathrm{H}, \mathrm{NH}), 11.15(\mathrm{~s}, 1 \mathrm{H}, \mathrm{NH}), 8.17(\mathrm{~d}$, $J=8.1 \mathrm{~Hz}, 2 \mathrm{H}, \mathrm{Ph}), 7.90(\mathrm{~d}, J=8.1 \mathrm{~Hz}, 2 \mathrm{H}, \mathrm{Ph}), 6.38(\mathrm{~s}, 1 \mathrm{H}, \mathrm{ArH}), 5.07\left(\mathrm{q}, J=8.9 \mathrm{~Hz}, 4 \mathrm{H}, \mathrm{OCH}_{2} \mathrm{CF}_{3}\right)$. ${ }^{13}$ C-NMR (DMSO- $d_{6}$ ) $\delta 170.9,167.9,156.4,149.3,137.4,133.7,133.5,130.1,126.5,126.5,125.8,125.6,86.5$, 63.7, 63.4, 63.1, 62.8. HRMS (ESI): $m / z$ calcd for $\mathrm{C}_{17} \mathrm{H}_{11} \mathrm{~F}_{9} \mathrm{~N}_{4} \mathrm{O}_{4}[\mathrm{M}+\mathrm{H}]^{+}:$507.0715, found 507.0713.

$N$-((4,6-bis(2,2,2-Trifluoroethoxy)pyrimidin-2-yl)carbamoyl)-5-chloro-2-methoxybenzamide (8). White solid, mp 164-166 ${ }^{\circ} \mathrm{C}$, yield $=95 \% .{ }^{1} \mathrm{H}-\mathrm{NMR}\left(\mathrm{DMSO}_{6}\right) \delta 11.04(\mathrm{~s}, 1 \mathrm{H}, \mathrm{NH}), 10.98(\mathrm{~s}, 1 \mathrm{H}, \mathrm{NH}), 7.65(\mathrm{~d}$, $J=2.7 \mathrm{~Hz}, 1 \mathrm{H}, \mathrm{Ph}), 7.61(\mathrm{dd}, J=8.9,2.8 \mathrm{~Hz}, 1 \mathrm{H}, \mathrm{Ph}), 7.23(\mathrm{~d}, J=8.9 \mathrm{~Hz}, 1 \mathrm{H}, \mathrm{Ph}), 6.39(\mathrm{~s}, 1 \mathrm{H}, \mathrm{ArH})$, $5.06\left(\mathrm{q}, J=8.9 \mathrm{~Hz}, 4 \mathrm{H}, \mathrm{OCH}_{2} \mathrm{CF}_{3}\right), 3.89\left(\mathrm{~s}, 3 \mathrm{H}, \mathrm{CH}_{3}\right) .{ }^{13} \mathrm{C}-\mathrm{NMR}\left(\mathrm{DMSO}-d_{6}\right) \delta 170.9,167.3,156.6,156.3$, 148.9, 133.9, 130.1, 125.4, 125.1, 115.3, 86.7, 63.5, 63.3, 63.0, 62.7, 57.5. HRMS (ESI): $m / z$ calcd for $\mathrm{C}_{17} \mathrm{H}_{13} \mathrm{ClF}_{6} \mathrm{~N}_{4} \mathrm{O}_{5}[\mathrm{M}+\mathrm{H}]^{+}$: 503.0557, found 503.0535.

$\mathrm{N}$-((4,6-bis(2,2,2-Trifluoroethoxy)pyrimidin-2-yl)carbamoyl)-2,4-dichlorobenzamide (9). White solid, $\mathrm{mp}$ 109-110 ${ }^{\circ} \mathrm{C}$, yield $=92 \% .{ }^{1} \mathrm{H}-\mathrm{NMR}\left(\mathrm{DMSO}_{6}\right) \delta 11.49(\mathrm{~s}, 1 \mathrm{H}, \mathrm{NH}), 10.88(\mathrm{~s}, 1 \mathrm{H}, \mathrm{NH}), 7.79(\mathrm{~d}, J=1.9 \mathrm{~Hz}$, $1 \mathrm{H}, \mathrm{Ph}), 7.68(\mathrm{~d}, J=8.2 \mathrm{~Hz}, 1 \mathrm{H}, \mathrm{Ph}), 7.58(\mathrm{dd}, J=8.2,2.0 \mathrm{~Hz}, 1 \mathrm{H}, \mathrm{Ph}), 6.40(\mathrm{~s}, 1 \mathrm{H}, \mathrm{ArH}), 5.06(\mathrm{q}$, $\left.J=8.9 \mathrm{~Hz}, 4 \mathrm{H}, \mathrm{OCH}_{2} \mathrm{CF}_{3}\right) .{ }^{13} \mathrm{C}-\mathrm{NMR}\left(\mathrm{DMSO}-d_{6}\right) \delta 170.8,168.3,156.3,149.0,136.9,134.6,132.0,131.4$, 130.3, 128.5, 125.6, 86.8, 63.6, 63.3, 63.0. HRMS (ESI): $m / z$ calcd for $\mathrm{C}_{16} \mathrm{H}_{10} \mathrm{Cl}_{2} \mathrm{~F}_{6} \mathrm{~N}_{4} \mathrm{O}_{4}[\mathrm{M}+\mathrm{Na}]^{+}$: 528.9881 , found 528.9866 .

$\mathrm{N}$-((4,6-bis(2,2,2-Trifluoroethoxy)pyrimidin-2-yl)carbamoyl)-2,6-dichlorobenzamide (10). White solid, $\mathrm{mp}$ $62-64{ }^{\circ} \mathrm{C}$, yield $=81 \% .{ }^{1} \mathrm{H}-\mathrm{NMR}\left(\mathrm{DMSO}_{6}\right) \delta 11.68(\mathrm{~s}, 1 \mathrm{H}, \mathrm{NH}), 10.80(\mathrm{~s}, 1 \mathrm{H}, \mathrm{NH}), 7.82-7.39(\mathrm{~m}, 3 \mathrm{H}$, $\mathrm{Ph}), 6.41(\mathrm{~s}, 1 \mathrm{H}, \mathrm{ArH}), 5.08\left(\mathrm{q}, J=8.9 \mathrm{~Hz}, 4 \mathrm{H}, \mathrm{OCH}_{2} \mathrm{CF}_{3}\right) .{ }^{13} \mathrm{C}-\mathrm{NMR}$ (DMSO- $\left.d_{6}\right) \delta 170.9,156.2,148.8$, 131.4, 129.2, 127.8, 125.6, 123.4, 121.2, 100.5, 86.9, 63.7, 63.4, 63.1, 62.9. HRMS (ESI): $m / z$ calcd for $\mathrm{C}_{16} \mathrm{H}_{10} \mathrm{Cl}_{2} \mathrm{~F}_{6} \mathrm{~N}_{4} \mathrm{O}_{4}[\mathrm{M}+\mathrm{Na}]^{+}$: 528.9881, found 528.9866.

2-Chloro-N-((4,6-dimethylpyrimidin-2-yl)carbamoyl)benzamide (11). White solid, $\mathrm{mp}$ 186-188 ${ }^{\circ} \mathrm{C}$, yield $=87 \% .{ }^{1} \mathrm{H}-\mathrm{NMR}\left(\mathrm{DMSO}-d_{6}\right) \delta 12.51(\mathrm{~s}, 1 \mathrm{H}, \mathrm{NH}), 10.61(\mathrm{~s}, 1 \mathrm{H}, \mathrm{NH}), 7.64(\mathrm{dd}, J=7.6,1.6 \mathrm{~Hz}, 1 \mathrm{H}$, $\mathrm{Ph})$, 7.61-7.52 (m, 2H, Ph), 7.47 (td, J = 7.3, 1.5 Hz, 1H, Ph), 6.98 (s, 1H, ArH), $2.33\left(\mathrm{~s}, 6 \mathrm{H}, \mathrm{Ar}_{-} \mathrm{CH}_{3}\right)$. ${ }^{13} \mathrm{C}-\mathrm{NMR}\left(\mathrm{DMSO}-d_{6}\right) \delta 168.8,167.5,157.6,149.8,136.3,132.9,130.8,130.6,129.8,128.3,116.0,24.2$. HRMS (ESI): $m / z$ calcd for $\mathrm{C}_{14} \mathrm{H}_{13} \mathrm{ClN}_{4} \mathrm{O}_{2}[\mathrm{M}+\mathrm{Na}]^{+}: 327.0625$, found 327.0629.

2-Chloro- $\mathrm{N}$-((4,6-dimethoxypyrimidin-2-yl)carbamoyl)benzamide (12). White solid, $\mathrm{mp} 155-157{ }^{\circ} \mathrm{C}$, yield $=89 \% .{ }^{1} \mathrm{H}-\mathrm{NMR}\left(\mathrm{DMSO}-d_{6}\right) \delta 11.87(\mathrm{~s}, 1 \mathrm{H}, \mathrm{NH}), 10.67(\mathrm{~s}, 1 \mathrm{H}, \mathrm{NH}), 7.62(\mathrm{dd}, J=7.6,1.6 \mathrm{~Hz}, 1 \mathrm{H}$, $\mathrm{Ph}), 7.59-7.50(\mathrm{~m}, 2 \mathrm{H}, \mathrm{Ph}), 7.46(\mathrm{td}, J=7.3,1.5 \mathrm{~Hz}, 1 \mathrm{H}, \mathrm{Ph}), 5.97(\mathrm{~s}, 1 \mathrm{H}, \mathrm{ArH}), 3.82\left(\mathrm{~s}, 6 \mathrm{H}, \mathrm{Ar}-\mathrm{OCH}_{3}\right)$. ${ }^{13} \mathrm{C}-\mathrm{NMR}$ (DMSO- $d_{6}$ ) $\delta 172.4,168.4,156.9,149.4,136.3,132.8,130.6,130.5,129.7,128.3,85.1,55.2$. HRMS (ESI): $m / z$ calcd for $\mathrm{C}_{14} \mathrm{H}_{13} \mathrm{ClN}_{4} \mathrm{O}_{4}[\mathrm{M}+\mathrm{Na}]^{+}:$359.0523, found 359.0524 .

2-Chloro-N-((4,6-diethoxypyrimidin-2-yl)carbamoyl)benzamide (13). White solid, mp 113-115 ${ }^{\circ} \mathrm{C}$, yield $=91 \% .{ }^{1} \mathrm{H}-\mathrm{NMR}\left(\mathrm{DMSO}-d_{6}\right) \delta 11.92(\mathrm{~s}, 1 \mathrm{H}, \mathrm{NH}), 10.61(\mathrm{~s}, 1 \mathrm{H}, \mathrm{NH}), 7.65(\mathrm{dd}, J=7.6,1.5 \mathrm{~Hz}, 1 \mathrm{H}$, $\mathrm{Ph}), 7.57$ (qd, $J=8.1,4.1 \mathrm{~Hz}, 2 \mathrm{H}, \mathrm{Ph}), 7.52-7.44(\mathrm{~m}, 1 \mathrm{H}, \mathrm{Ph}), 5.87(\mathrm{~s}, 1 \mathrm{H}, \mathrm{ArH}), 4.20(\mathrm{q}, J=7.0 \mathrm{~Hz}, 4 \mathrm{H}$, $\left.\mathrm{OCH}_{2} \mathrm{CH}_{3}\right), 1.26\left(\mathrm{t}, J=7.1 \mathrm{~Hz}, 6 \mathrm{H}, \mathrm{OCH}_{2} \mathrm{CH}_{3}\right) .{ }^{13} \mathrm{C}-\mathrm{NMR}\left(\mathrm{DMSO}-d_{6}\right) \delta 171.9,167.9,156.9,149.4,136.3$, 135.8, 132.8, 130.6, 129.7, 128.3, 85.3, 64.5, 63.6, 15.2, 15.0. HRMS (ESI): $m / z$ calcd for $\mathrm{C}_{16} \mathrm{H}_{17} \mathrm{ClN}_{4} \mathrm{O}_{4}$ $[\mathrm{M}+\mathrm{Na}]^{+}: 387.0836$, found 387.0851 . 
2-Chloro-N-((4,6-dichloropyrimidin-2-yl)carbamoyl)benzamide (14). White solid, $\mathrm{mp} 156-158{ }^{\circ} \mathrm{C}$, yield $=88 \% .{ }^{1} \mathrm{H}-\mathrm{NMR}\left(\mathrm{DMSO}-d_{6}\right) \delta 11.52(\mathrm{~s}, 1 \mathrm{H}, \mathrm{NH}), 11.24(\mathrm{~s}, 1 \mathrm{H}, \mathrm{NH}), 7.69$ (s, 1H, ArH), 7.65 (dd, $J=7.6,1.5 \mathrm{~Hz}, 1 \mathrm{H}, \mathrm{Ph}), 7.57$ (qd, $J=8.1,1.7 \mathrm{~Hz}, 2 \mathrm{H}, \mathrm{Ph}), 7.49$ (td, $J=7.2,1.9 \mathrm{~Hz}, 1 \mathrm{H}, \mathrm{Ph}) .{ }^{13} \mathrm{C}-\mathrm{NMR}$ $\left(\right.$ DMSO- $\left.d_{6}\right) \delta 169.0,162.5,157.4,148.8,133.1,130.8,130.8,130.0,128.3,117.0$. HRMS (ESI): $\mathrm{m} / z$ calcd for $\mathrm{C}_{12} \mathrm{H}_{7} \mathrm{Cl}_{3} \mathrm{~N}_{4} \mathrm{O}_{2}[\mathrm{M}+\mathrm{Na}]^{+}: 366.9532$, found 366.9549 .

2-Chloro-N-((4-chloro-6-methylpyrimidin-2-yl)carbamoyl)benzamide (15). White solid, mp $126-128{ }^{\circ} \mathrm{C}$, yield $=93 \% .{ }^{1} \mathrm{H}-\mathrm{NMR}\left(\mathrm{DMSO}-d_{6}\right) \delta 11.89(\mathrm{~s}, 1 \mathrm{H}, \mathrm{NH}), 10.98(\mathrm{~s}, 1 \mathrm{H}, \mathrm{NH}), 7.65(\mathrm{dd}, J=7.6,1.6 \mathrm{~Hz}$, 1H, Ph), 7.63-7.51 (m, 2H, Ph), $7.47(\mathrm{td}, J=7.3,1.5 \mathrm{~Hz}, 1 \mathrm{H}, \mathrm{Ph}), 7.32$ (s, 1H, ArH), $2.40\left(\mathrm{~s}, 3 \mathrm{H}, \mathrm{CH}_{3}\right)$. ${ }^{13}$ C-NMR (DMSO-d $\left.d_{6}\right) \delta 171.4,169.1,168.3,161.4,157.5,149.2,138.1,131.5,130.5,129.6,127.9,116.4,24.2$. HRMS (ESI): $m / z$ calcd for $\mathrm{C}_{13} \mathrm{H}_{10} \mathrm{Cl}_{2} \mathrm{~N}_{4} \mathrm{O}_{2}[\mathrm{M}+\mathrm{Na}]^{+}: 347.0079$, found 347.0064 .

2-Chloro-N-((4-chloro-6-methoxypyrimidin-2-yl)carbamoyl)benzamide (16). White solid, mp $166-167{ }^{\circ} \mathrm{C}$, yield $=87 \% .{ }^{1} \mathrm{H}-\mathrm{NMR}\left(\mathrm{DMSO}-d_{6}\right) \delta 11.64(\mathrm{~s}, 1 \mathrm{H}, \mathrm{NH}), 10.98(\mathrm{~s}, 1 \mathrm{H}, \mathrm{NH}), 7.65(\mathrm{~d}, J=7.5 \mathrm{~Hz}, 1 \mathrm{H}, \mathrm{Ph})$, $7.56(\mathrm{~d}, J=8.5 \mathrm{~Hz}, 2 \mathrm{H}, \mathrm{Ph}), 7.48(\mathrm{t}, J=7.3 \mathrm{~Hz}, 1 \mathrm{H}, \mathrm{Ph}), 6.82(\mathrm{~s}, 1 \mathrm{H}, \mathrm{ArH}), 3.91\left(\mathrm{~s}, 3 \mathrm{H}, \mathrm{OCH}_{3}\right) .{ }^{13} \mathrm{C}-\mathrm{NMR}$ $\left(\right.$ DMSO-d $\left.d_{6}\right) \delta 171.9,168.6,160.8,157.2,149.0,135.7,132.9,130.6,129.9,128.2,102.6,55.7$. HRMS (ESI): $m / z$ calcd for $\mathrm{C}_{13} \mathrm{H}_{10} \mathrm{Cl}_{2} \mathrm{~N}_{4} \mathrm{O}_{3}[\mathrm{M}+\mathrm{Na}]^{+}$: 363.0028, found 363.0013.

2-Chloro-N-((4-chloro-6-(2,2,2-trifluoroethoxy)pyrimidin-2-yl)carbamoyl)benzamide (17). White solid, $\mathrm{mp}$ 144-146 ${ }^{\circ} \mathrm{C}$, yield = 88\%. ${ }^{1} \mathrm{H}-\mathrm{NMR}\left(\mathrm{DMSO}_{6}\right) \delta 11.51(\mathrm{~s}, 1 \mathrm{H}, \mathrm{NH}), 11.07(\mathrm{~s}, 1 \mathrm{H}, \mathrm{NH}), 7.65(\mathrm{dd}, J=7.5$, $1.5 \mathrm{~Hz}, 1 \mathrm{H}, \mathrm{Ph}), 7.62-7.51(\mathrm{~m}, 2 \mathrm{H}, \mathrm{Ph}), 7.48$ (td, $J=7.3,1.7 \mathrm{~Hz}, 1 \mathrm{H}, \mathrm{Ph}), 7.09$ (s, 1H, ArH), $5.10(\mathrm{q}$, $\left.J=8.8 \mathrm{~Hz}, 2 \mathrm{H}, \mathrm{OCH}_{2} \mathrm{CF}_{3}\right) .{ }^{13} \mathrm{C}-\mathrm{NMR}\left(\mathrm{DMSO}-d_{6}\right) \delta 170.0,169.1,161.8,156.9,148.9,135.6,133.1,130.8$, 130.0, 128.2, 125.5, 123.3, 103.0, 63.8, 63.5, 63.2, 62.9. HRMS (ESI): $m / z$ calcd for $\mathrm{C}_{14} \mathrm{H}_{9} \mathrm{Cl}_{2} \mathrm{~F}_{3} \mathrm{~N}_{4} \mathrm{O}_{3}$ $[\mathrm{M}+\mathrm{Na}]^{+}:$430.9901, found 430.9887 .

2-Chloro-N-((4-chloro-6-morpholinopyrimidin-2-yl)carbamoyl)benzamide (18). White solid, mp $147-149{ }^{\circ} \mathrm{C}$, yield $=85 \% .{ }^{1} \mathrm{H}-\mathrm{NMR}\left(\mathrm{DMSO}-d_{6}\right) \delta 11.78(\mathrm{~s}, 1 \mathrm{H}, \mathrm{NH}), 10.59(\mathrm{~s}, 1 \mathrm{H}, \mathrm{NH}), 7.62(\mathrm{dd}, J=7.6,1.6 \mathrm{~Hz}, 1 \mathrm{H}$, $\mathrm{Ph}$ ), 7.59-7.50 (m, 2H, Ph), 7.46 (td, $J=7.3,1.5 \mathrm{~Hz}, 1 \mathrm{H}, \mathrm{Ph}), 6.71$ (s, 1H, ArH), 3.58 (dd, J = 23.7, 4.9 Hz, 8H, morpholine). ${ }^{13} \mathrm{C}-\mathrm{NMR}$ (DMSO- $\left.d_{6}\right) \delta 168.2,163.6,157.0,149.3,136.1,132.9,130.7,130.6,129.8$, 128.3, 97.9, 66.6, 60.7. HRMS (ESI): $m / z$ calcd for $\mathrm{C}_{16} \mathrm{H}_{15} \mathrm{Cl}_{2} \mathrm{~N}_{5} \mathrm{O}_{3}[\mathrm{M}+\mathrm{Na}]^{+}:$418.0450, found 418.0441 .

2-Chloro-N-((4-chloro-6-(diethylamino)pyrimidin-2-yl)carbamoyl)benzamide (19). white solid, mp 151-153 ${ }^{\circ} \mathrm{C}$, yield = 83\%. ${ }^{1} \mathrm{H}-\mathrm{NMR}\left(\mathrm{DMSO}_{6}\right) \delta 11.97(\mathrm{~s}, 1 \mathrm{H}, \mathrm{NH}), 10.46(\mathrm{~s}, 1 \mathrm{H}, \mathrm{NH}), 7.60$ (dd, $J=7.6,1.6 \mathrm{~Hz}, 1 \mathrm{H}, \mathrm{Ph}), 7.58-7.49$ (m, 2H, Ph), $7.45(\mathrm{td}, J=7.3,1.6 \mathrm{~Hz}, 1 \mathrm{H}, \mathrm{Ph}), 6.49$ (s, 1H, ArH), 3.44 $\left(\mathrm{s}, 4 \mathrm{H}, \mathrm{CH}_{2} \mathrm{CH}_{3}\right), 1.19-0.91\left(\mathrm{~m}, 6 \mathrm{H}, \mathrm{CH}_{2} \mathrm{CH}_{3}\right) .{ }^{13} \mathrm{C}-\mathrm{NMR}\left(\mathrm{DMSO}-d_{6}\right) \delta 168.1,162.1,159.4,157.2,149.6$, 136.5, 132.7, 130.5, 130.5, 129.7, 128.3, 97.1, 43.3, 13.4. HRMS (ESI): $m / z$ calcd for $\mathrm{C}_{16} \mathrm{H}_{17} \mathrm{Cl}_{2} \mathrm{~N}_{5} \mathrm{O}_{2}[\mathrm{M}+$ $\mathrm{Na}]^{+}:$404.0657, found 404.0642.

2-Chloro-N-((4-methyl-6-(2,2,2-trifluoroethoxy)pyrimidin-2-yl)carbamoyl)benzamide (20). White solid, $\mathrm{mp}$ 56-58 ${ }^{\circ} \mathrm{C}$, yield $=82 \% .{ }^{1} \mathrm{H}-\mathrm{NMR}\left(\mathrm{DMSO}_{6}\right) \delta 12.13(\mathrm{~s}, 1 \mathrm{H}, \mathrm{NH}), 10.79(\mathrm{~s}, 1 \mathrm{H}, \mathrm{NH}), 7.72-7.63(\mathrm{~m}, 1 \mathrm{H}$, $\mathrm{Ph}), 7.63-7.52(\mathrm{~m}, 2 \mathrm{H}, \mathrm{Ph}), 7.49(\mathrm{td}, J=7.3,1.6 \mathrm{~Hz}, 1 \mathrm{H}, \mathrm{Ph}), 6.71(\mathrm{~s}, 1 \mathrm{H}, \mathrm{ArH}), 5.07(\mathrm{q}, J=8.9 \mathrm{~Hz}, 2 \mathrm{H}$, $\left.\mathrm{OCH}_{2} \mathrm{CF}_{3}\right), 2.34\left(\mathrm{~s}, 3 \mathrm{H}, \mathrm{CH}_{3}\right) .{ }^{13} \mathrm{C}-\mathrm{NMR}\left(\mathrm{DMSO}-d_{6}\right) \delta 171.4,170.3,169.3,168.1,161.4,157.0,149.4,136.0$, $132.9,130.7,129.9,128.2,102.1,62.8,62.5,62.2,61.9,24.1$. HRMS (ESI): $m / z$ calcd for $\mathrm{C}_{15} \mathrm{H}_{12} \mathrm{ClF}_{3} \mathrm{~N}_{4} \mathrm{O}_{3}$ $[\mathrm{M}+\mathrm{Na}]^{+}:$411.0448, found 411.0442 .

2-Chloro-N-((4-(pyrrolidin-1-yl)-6-(2,2,2-trifluoroethoxy)pyrimidin-2-yl)carbamoyl)benzamide (21). White solid, mp $121-122{ }^{\circ} \mathrm{C}$, yield $=81 \% .{ }^{1} \mathrm{H}-\mathrm{NMR}\left(\mathrm{DMSO}-d_{6}\right) \delta 12.55(\mathrm{~s}, 1 \mathrm{H}, \mathrm{NH}), 10.28(\mathrm{~s}, 1 \mathrm{H}, \mathrm{NH}), 7.61(\mathrm{~d}$, $J=7.4 \mathrm{~Hz}, 1 \mathrm{H}, \mathrm{Ph}), 7.58-7.50(\mathrm{~m}, 2 \mathrm{H}, \mathrm{Ph}), 7.46$ (td, J = 7.3, 1.7 Hz, 1H, Ph), 5.62 (s, 1H, ArH), 4.97 (q, $\left.J=9.0 \mathrm{~Hz}, 2 \mathrm{H}, \mathrm{OCH}_{2} \mathrm{CF}_{3}\right), 3.25$ (s, 2H, tetrahydropyrrole), 3.02 (s, 2H, tetrahydropyrrole), 1.97-1.82 (m, 2H, tetrahydropyrrole), 1.77-1.60 (m, 2H, tetrahydropyrrole). ${ }^{13} \mathrm{C}-\mathrm{NMR}$ (DMSO- $\left.d_{6}\right) \delta 168.7,167.3$, $161.8,156.7,149.8,136.9,132.5,130.5,130.3,129.4,128.3,125.9,81.1,62.2,61.9,61.6,47.3,25.7$. HRMS (ESI): $m / z$ calcd for $\mathrm{C}_{18} \mathrm{H}_{17} \mathrm{ClF}_{3} \mathrm{~N}_{5} \mathrm{O}_{3}[\mathrm{M}+\mathrm{H}]^{+}: 444.1050$, found 444.1047 .

2-Chloro-N-((4-(hexylamino)-6-(2,2,2-trifluoroethoxy)pyrimidin-2-yl)carbamoyl)benzamide (22). White solid, mp $126-128{ }^{\circ} \mathrm{C}$, yield $=83 \% .{ }^{1} \mathrm{H}-\mathrm{NMR}\left(\mathrm{DMSO}-d_{6}\right) \delta 12.40(\mathrm{~s}, 1 \mathrm{H}, \mathrm{NH}), 10.28(\mathrm{~s}, 1 \mathrm{H}, \mathrm{NH}), 7.85-7.36(\mathrm{~m}$, 
$5 \mathrm{H}, \mathrm{Ph}), 5.62(\mathrm{~s}, 1 \mathrm{H}, \mathrm{ArH}), 4.93\left(\mathrm{q}, J=9.0 \mathrm{~Hz}, 2 \mathrm{H}, \mathrm{OCH}_{2} \mathrm{CF}_{3}\right), 3.05\left(\mathrm{~s}, 2 \mathrm{H}, \mathrm{N}-\mathrm{CH}_{2}\left(\mathrm{CH}_{2}\right)_{4} \mathrm{CH}_{3}\right), 1.46-1.07$ $\left(\mathrm{m}, 8 \mathrm{H}, \mathrm{N}-\mathrm{CH}_{2}\left(\mathrm{CH}_{2}\right)_{4} \mathrm{CH}_{3}\right), 0.86\left(\mathrm{t}, J=6.8 \mathrm{~Hz}, 3 \mathrm{H}, \mathrm{N}\left(\mathrm{CH}_{2}\right)_{5} \mathrm{CH}_{3}\right) .{ }^{13} \mathrm{C}-\mathrm{NMR}$ (DMSO-d 6 ) $\delta 168.0,165.1$, 157.1, 151.8, 149.8, 136.7, 132.6, 130.5, 129.6, 128.3, 125.9, 123.6, 82.2, 62.1, 61.8, 41.3, 32.0, 29.6, 27.0, 23.1, 14.9. HRMS (ESI): $m / z$ calcd for $\mathrm{C}_{20} \mathrm{H}_{23} \mathrm{ClF}_{3} \mathrm{~N}_{5} \mathrm{O}_{3}[\mathrm{M}+\mathrm{Na}]^{+}: 496.1339$, found 496.1357 .

2,4-Dichloro- $\mathrm{N}$-((4,6-dimethylpyrimidin-2-yl)carbamoyl)benzamide (23). White solid, mp $185-186{ }^{\circ} \mathrm{C}$, yield $=81 \% .{ }^{1} \mathrm{H}-\mathrm{NMR}\left(\mathrm{DMSO}_{6}\right) \delta 12.50(\mathrm{~s}, 1 \mathrm{H}, \mathrm{NH}), 10.60(\mathrm{~s}, 1 \mathrm{H}, \mathrm{NH}), 7.77(\mathrm{~d}, J=2.0 \mathrm{~Hz}, 1 \mathrm{H}$, $\mathrm{Ph}), 7.67(\mathrm{~d}, J=8.2 \mathrm{~Hz}, 1 \mathrm{H}, \mathrm{Ph}), 7.57(\mathrm{dd}, J=8.2,2.0 \mathrm{~Hz}, 1 \mathrm{H}, \mathrm{Ph}), 6.99(\mathrm{~d}, J=2.7 \mathrm{~Hz}, 1 \mathrm{H}, \mathrm{ArH}), 2.35$ (s, $6 \mathrm{H}, \mathrm{CH}_{3}$ ). ${ }^{13} \mathrm{C}-\mathrm{NMR}$ (DMSO- $d_{6}$ ) $\delta 168.8,167.0,157.6,149.8,136.5,135.4,131.8,131.1,130.3,128.5,116.1$, 24.3. HRMS (ESI): $m / z$ calcd for $\mathrm{C}_{14} \mathrm{H}_{12} \mathrm{Cl}_{2} \mathrm{~N}_{4} \mathrm{O}_{2}[\mathrm{M}+\mathrm{Na}]^{+}: 361.0235$, found 361.0208 .

2,4-Dichloro-N-((4-methyl-6-(2,2,2-trifluoroethoxy)pyrimidin-2-yl)carbamoyl)benzamide (24). White solid, mp $155-157{ }^{\circ} \mathrm{C}$, yield $=81 \% .{ }^{1} \mathrm{H}-\mathrm{NMR}\left(\mathrm{DMSO}-d_{6}\right) \delta 12.14(\mathrm{~s}, 1 \mathrm{H}, \mathrm{NH}), 10.77(\mathrm{~s}, 1 \mathrm{H}, \mathrm{NH}), 7.76(\mathrm{~d}$, $J=2.0 \mathrm{~Hz}, 1 \mathrm{H}, \mathrm{Ph}), 7.70(\mathrm{~d}, J=8.3 \mathrm{~Hz}, 1 \mathrm{H}, \mathrm{Ph}), 7.64-7.54(\mathrm{~m}, 1 \mathrm{H}, \mathrm{Ph}), 6.71(\mathrm{~s}, 1 \mathrm{H}, \mathrm{ArH}), 5.07(\mathrm{q}$, $\left.J=8.9 \mathrm{~Hz}, 2 \mathrm{H}, \mathrm{OCH}_{2} \mathrm{CF}_{3}\right), 2.37\left(\mathrm{~s}, 3 \mathrm{H}, \mathrm{CH}_{3}\right) .{ }^{13} \mathrm{C}-\mathrm{NMR}\left(\mathrm{DMSO}-d_{6}\right) \delta 170.4,169.3,167.5,157.1,149.5$, 136.7, 135.0, 132.0, 131.2, 130.3, 128.5, 125.7, 102.2, 62.9, 62.6, 62.3, 62.0, 24.2. HRMS (ESI): $\mathrm{m} / \mathrm{z}$ calcd for $\mathrm{C}_{15} \mathrm{H}_{11} \mathrm{Cl}_{2} \mathrm{~F}_{3} \mathrm{~N}_{4} \mathrm{O}_{3}[\mathrm{M}+\mathrm{Na}]^{+}$: 445.0058, found 445.0064.

2,6-Dichloro-N-((4-chloro-6-(2,2,2-trifluoroethoxy)pyrimidin-2-yl)carbamoyl)benzamide (25). White solid, mp $125-126{ }^{\circ} \mathrm{C}$, yield $=87 \% .{ }^{1} \mathrm{H}-\mathrm{NMR}\left(-\mathrm{DMSO}-d_{6}\right) \delta 11.64(\mathrm{~s}, 1 \mathrm{H}, \mathrm{NH}), 10.90(\mathrm{~s}, 1 \mathrm{H}, \mathrm{NH}), 7.59$ $(\mathrm{d}, J=8.9 \mathrm{~Hz}, 2 \mathrm{H}, \mathrm{Ph}), 7.56-7.48(\mathrm{~m}, 1 \mathrm{H}, \mathrm{Ph}), 7.12(\mathrm{~s}, 1 \mathrm{H}, \mathrm{ArH}), 5.11\left(\mathrm{q}, J=8.9 \mathrm{~Hz}, 2 \mathrm{H}, \mathrm{OCH}_{2} \mathrm{CF}_{3}\right)$. ${ }^{13}$ C-NMR (DMSO- $d_{6}$ ) $\delta$ 170.0, 165.0, 161.8, 156.8, 148.7, 137.7, 132.1, 131.9, 129.0, 125.4, 103.2, 63.6, 63.3, 63.0. HRMS (ESI): $m / z$ calcd for $\mathrm{C}_{14} \mathrm{H}_{8} \mathrm{Cl}_{3} \mathrm{~F}_{3} \mathrm{~N}_{4} \mathrm{O}_{3}[\mathrm{M}+\mathrm{Na}]^{+}: 464.9512$, found 464.9502 .

2,6-Dichloro-N-((4-chloro-6-(diethylamino)pyrimidin-2-yl)carbamoyl)benzamide (26). White solid, mp 169-171 ${ }^{\circ} \mathrm{C}$, yield $=81 \% .{ }^{1} \mathrm{H}-\mathrm{NMR}\left(\mathrm{DMSO}-d_{6}\right) \delta 12.42(\mathrm{~s}, 1 \mathrm{H}, \mathrm{NH}), 10.44(\mathrm{~s}, 1 \mathrm{H}, \mathrm{NH}), 7.61-7.47$ $(\mathrm{m}, 3 \mathrm{H}, \mathrm{Ph}), 6.54(\mathrm{~s}, 1 \mathrm{H}, \mathrm{ArH}), 3.46\left(\mathrm{~s}, 4 \mathrm{H}, \mathrm{CH}_{2} \mathrm{CH}_{3}\right), 1.12\left(\mathrm{~s}, 6 \mathrm{H}, \mathrm{CH}_{2} \mathrm{CH}_{3}\right) .{ }^{13} \mathrm{C}-\mathrm{NMR}\left(\mathrm{DMSO}-d_{6}\right) \delta$ 165.0, 164.0, 160.9, 158.5, 136.7, 131.1, 130.9, 128.1, 96.2, 42.6, 25.8, 12.4. HRMS (ESI): $\mathrm{m} / \mathrm{z}$ calcd for $\mathrm{C}_{16} \mathrm{H}_{16} \mathrm{Cl}_{3} \mathrm{~N}_{5} \mathrm{O}_{2}[\mathrm{M}+\mathrm{H}]^{+}:$416.0448, found 416.0444.

2,6-Dichloro-N-((4-(diethylamino)-6-(2,2,2-trifluoroethoxy)pyrimidin-2-yl)carbamoyl)benzamide (27). White solid, mp 140-142 ${ }^{\circ} \mathrm{C}$, yield $=87 \% .{ }^{1} \mathrm{H}-\mathrm{NMR}\left(\right.$ DMSO- $\left._{6}\right) \delta 12.84(\mathrm{~s}, 1 \mathrm{H}, \mathrm{NH}), 10.22(\mathrm{~s}, 1 \mathrm{H}, \mathrm{NH})$, 8.60-6.40 (m, 3H, Ph), $5.84(\mathrm{~s}, 1 \mathrm{H}, \mathrm{ArH}), 4.96\left(\mathrm{q}, J=9.0 \mathrm{~Hz}, 2 \mathrm{H}, \mathrm{OCH}_{2} \mathrm{CF}_{3}\right), 3.46\left(\mathrm{~s}, 4 \mathrm{H}, \mathrm{CH}_{2} \mathrm{CH}_{3}\right), 1.12$ (s, 6H, $\mathrm{CH}_{2} \mathrm{CH}_{3}$ ). ${ }^{13} \mathrm{C}-\mathrm{NMR}$ (DMSO- $d_{6}$ ) $\delta 169.2,168.2,163.3,156.7,149.8,138.1,131.5,130.6,129.7$, 127.9, 80.4, 62.6, 62.3, 62.0, 61.7, 43.2, 13.4. HRMS (ESI): $m / z$ calcd for $\mathrm{C}_{18} \mathrm{H}_{18} \mathrm{Cl}_{2} \mathrm{~F}_{3} \mathrm{~N}_{5} \mathrm{O}_{3}[\mathrm{M}+\mathrm{Na}]^{+}$: 502.0636 , found 502.0635 .

N-((4-(Benzylamino)-6-(2,2,2-trifluoroethoxy)pyrimidin-2-yl)carbamoyl)-2,6-dichlorobenzamide (28). White solid, mp 76-78 ${ }^{\circ} \mathrm{C}$, yield $=80 \%$. ${ }^{1} \mathrm{H}-\mathrm{NMR}\left(\mathrm{DMSO}_{6}\right) \delta 12.46(\mathrm{~d}, J=369.0 \mathrm{~Hz}, 1 \mathrm{H}, \mathrm{NH}), 10.27(\mathrm{~s}$, $1 \mathrm{H}, \mathrm{NH}), 8.25$ (s, 1H, Ar-NH), 7.82-7.40 (m, 3H, Ph), 7.42-7.14 (m, 5H, Ph), 5.70 (s, 1H, ArH), 4.93 (q, $\left.J=9.0 \mathrm{~Hz}, 2 \mathrm{H}, \mathrm{OCH}_{2} \mathrm{CF}_{3}\right), 4.39\left(\mathrm{~s}, 2 \mathrm{H}, \mathrm{N}-\mathrm{CH}_{2} \mathrm{Ph}\right) .{ }^{13} \mathrm{C}-\mathrm{NMR}\left(\mathrm{DMSO}-d_{6}\right) \delta 164.0,155.9,151.0,148.6$, 138.7, 136.7, 131.1, 130.8, 129.9, 128.3, 128.0, 127.9, 127.0, 126.1, 124.8, 122.6, 120.4, 81.6, 61.6, 61.3, 61.0, 60.7, 44.2, 30.6, 25.7. HRMS (ESI): $m / z$ calcd for $\mathrm{C}_{21} \mathrm{H}_{16} \mathrm{Cl}_{2} \mathrm{~F}_{3} \mathrm{~N}_{5} \mathrm{O}_{3}[\mathrm{M}+\mathrm{Na}]^{+}: 536.0480$, found 536.0485 .

N-((4-(Benzyl(ethyl)amino)-6-(2,2,2-trifluoroethoxy)pyrimidin-2-yl)carbamoyl)-2,6-dichlorobenzamide (29). White solid, mp 61-62 ${ }^{\circ} \mathrm{C}$, yield $=75 \% .{ }^{1} \mathrm{H}-\mathrm{NMR}\left(\mathrm{DMSO}-d_{6}\right) \delta 12.72(\mathrm{~s}, 1 \mathrm{H}, \mathrm{NH}), 10.28(\mathrm{~s}, 1 \mathrm{H}, \mathrm{NH})$, 7.71-7.08 (m, 8H, Ph), $5.85(\mathrm{~s}, 1 \mathrm{H}, \mathrm{ArH}), 4.95\left(\mathrm{~d}, J=9.0 \mathrm{~Hz}, 2 \mathrm{H}, \mathrm{OCH}_{2} \mathrm{CF}_{3}\right), 4.74\left(\mathrm{~s}, 2 \mathrm{H}, \mathrm{N}-\mathrm{CH}_{2} \mathrm{Ph}\right), 3.41$ $\left(\mathrm{d}, J=52.6 \mathrm{~Hz}, 2 \mathrm{H}, \mathrm{CH}_{2} \mathrm{CH}_{3}\right), 1.08\left(\mathrm{~s}, 3 \mathrm{H}, \mathrm{CH}_{2} \mathrm{CH}_{3}\right) .{ }^{13} \mathrm{C}-\mathrm{NMR}$ (DMSO-d 6 ) $\delta 169.3,167.5,166.0,165.2$, 164.7, 156.6, 156.3, 133.7, 131.1, 129.8, 129.5, 129.0, 128.9, 128.1, 125.8, 123.6, 81.1, 62.5, 62.2, 61.9, 60.7, 30.9, 15.0. HRMS (ESI): $\mathrm{m} / z$ calcd for $\mathrm{C}_{23} \mathrm{H}_{20} \mathrm{Cl}_{2} \mathrm{~F}_{3} \mathrm{~N}_{5} \mathrm{O}_{3}[\mathrm{M}+\mathrm{H}]^{+}: 542.0974$, found 542.0971 .

$\mathrm{N}$-((5-(Sec-butyl)-4-chloro-6-(2,2,2-trifluoroethoxy)pyrimidin-2-yl)carbamoyl)-2,6-dichlorobenzamide (30). White solid, $\mathrm{mp} 37-38{ }^{\circ} \mathrm{C}$, yield $=73 \%$. ${ }^{1} \mathrm{H}-\mathrm{NMR}\left(\right.$ DMSO- $\left.d_{6}\right) \delta 11.64(\mathrm{~s}, 1 \mathrm{H}, \mathrm{NH}), 10.81(\mathrm{~s}, 1 \mathrm{H}$, $\mathrm{NH}), 7.76-7.39(\mathrm{~m}, 3 \mathrm{H}, \mathrm{Ph}), 5.16\left(\mathrm{q}, J=8.8 \mathrm{~Hz}, 2 \mathrm{H}, \mathrm{OCH}_{2} \mathrm{CF}_{3}\right), 3.21(\mathrm{dt}, J=9.2,6.8 \mathrm{~Hz}, 1 \mathrm{H}, \mathrm{CH})$, 
1.90-1.74 (m, $\left.1 \mathrm{H}, \mathrm{CHCH}_{2} \mathrm{CH}_{3}\right), 1.68\left(\mathrm{dt}, J=13.8,6.7 \mathrm{~Hz}, 1 \mathrm{H}, \mathrm{CHCH}_{2} \mathrm{CH}_{3}\right), 1.29(\mathrm{~d}, J=7.1 \mathrm{~Hz}, 3 \mathrm{H}$, $\left.\mathrm{CHCH}_{3}\right), 0.83\left(\mathrm{t}, J=7.4 \mathrm{~Hz}, 3 \mathrm{H}, \mathrm{CHCH}_{2} \mathrm{CH}_{3}\right) .{ }^{13} \mathrm{C}-\mathrm{NMR}\left(\mathrm{DMSO}-d_{6}\right) \delta 167.7,166.7,160.4,154.0,148.7$, 131.2, 129.1, 125.6, 123.4, 118.3, 63.9, 63.6, 63.3, 63.0, 31.6, 27.4, 18.6, 13.2. HRMS (ESI): $m / z$ calcd for $\mathrm{C}_{18} \mathrm{H}_{16} \mathrm{Cl}_{3} \mathrm{~F}_{3} \mathrm{~N}_{4} \mathrm{O}_{3}[\mathrm{M}+\mathrm{Na}]^{+}$: 521.0138, found 521.0157.

\subsection{Synthesis of $N-((4,6-b i s(2,2,2-T r i f l u o r o e t h o x y)$ pyrimidin-2-yl)carbamothioyl)-2-chlorobenzamide (31)}

Compound 31 was prepared by the method given in [22]. 2-Chlorobenzoic acid (5 mmol) and thionyl chloride $(10 \mathrm{~mL})$ were added to a $100 \mathrm{~mL}$ three-necked flask under $\mathrm{N}_{2}$. The reaction mixture was refluxed for $3 \mathrm{~h}$. The excess thionyl chloride was evaporated under reduced pressure to give a colorless transparent liquid. Anhydrous acetonitrile $(5 \mathrm{~mL})$ was added to the residue, then KSCN $(5 \mathrm{mmol})$ was added to the system and reacted at $80^{\circ} \mathrm{C}$ for $1 \mathrm{~h}$. The reaction mixture was cooled to the room temperature, filtered and neutralized with triethylamine. Then intermediate Ia $(5 \mathrm{mmol})$ and TBAB $(10 \mathrm{mmol})$ were added into reaction mixture. The mixture was heated to reflux for $4 \mathrm{~h}$. When the reaction was completed, the reaction mixture was cooled to room temperature, diluted with $20 \mathrm{~mL}$ of EtOAc, washed twice with brine $(20 \mathrm{~mL})$, dried with anhydrous $\mathrm{Na}_{2} \mathrm{SO}_{4}$ and evaporated in vacuo. Finally, the residue was purified by silica gel column chromatography to afford compound $\mathbf{3 1}$ as a yellow solid, mp 93-95 ${ }^{\circ} \mathrm{C}$, yield $=53 \% .{ }^{1} \mathrm{H}-\mathrm{NMR}\left(\mathrm{DMSO}_{6} d_{6}\right) \delta 12.84(\mathrm{~s}, 1 \mathrm{H}, \mathrm{NH}), 12.45(\mathrm{~s}, 1 \mathrm{H}, \mathrm{NH})$, 7.71-7.62 (m, 1H, Ph), 7.56 (dd, J = 6.5, 1.7 Hz, 2H, Ph), 7.51-7.42 (m, 1H, Ph), $6.51(\mathrm{~s}, 1 \mathrm{H}, \mathrm{ArH}), 5.11$ $\left(\mathrm{q}, J=8.9 \mathrm{~Hz}, 4 \mathrm{H}, \mathrm{OCH}_{2} \mathrm{CF}_{3}\right) .{ }^{13} \mathrm{C}-\mathrm{NMR}\left(\mathrm{DMSO}-d_{6}\right) \delta 178.3,170.8,168.3,156.2,135.2,133.3,131.1$, $130.7,130.3,128.2,125.6,123.4,88.2,63.9,63.6,63.3,63.0$. HRMS (ESI): $m / z$ calcd for $\mathrm{C}_{16} \mathrm{H}_{11} \mathrm{ClF}_{6} \mathrm{~N}_{4} \mathrm{O}_{3} \mathrm{~S}$ $[\mathrm{M}+\mathrm{Na}]^{+}:$511.0042, found 511.0015.

3.11. Synthesis of 2,6-Dichloro-N-((4-(diethylamino)-6-(2,2,2-trifluoroethoxy)pyrimidin-2-yl)carbamothioyl)benzamide (32a)

Compound 32a was synthesized by a method similar to that used for compound 31. Yellow solid, $\mathrm{mp} 42-43{ }^{\circ} \mathrm{C}$, yield $=59 \% .{ }^{1} \mathrm{H}-\mathrm{NMR}\left(\mathrm{DMSO}-d_{6}\right) \delta 12.84(\mathrm{~s}, 1 \mathrm{H}, \mathrm{NH}), 10.22(\mathrm{~s}, 1 \mathrm{H}, \mathrm{NH}), 7.93-7.14$ $(\mathrm{m}, 3 \mathrm{H}, \mathrm{Ph}), 5.84(\mathrm{~s}, 1 \mathrm{H}, \mathrm{ArH}), 4.96\left(\mathrm{q}, J=9.0 \mathrm{~Hz}, 2 \mathrm{H}, \mathrm{OCH}_{2} \mathrm{CF}_{3}\right), 3.46\left(\mathrm{~s}, 4 \mathrm{H}, \mathrm{CH}_{2} \mathrm{CH}_{3}\right), 1.12(\mathrm{~s}, 6 \mathrm{H}$, $\mathrm{CH}_{2} \mathrm{CH}_{3}$ ). ${ }^{13} \mathrm{C}-\mathrm{NMR}$ (DMSO- $d_{6}$ ) $\delta 169.2,168.2,163.2,156.7,149.8,132.5,131.5,130.6,129.7,127.9,80.4$, 62.6, 62.3, 62.0, 61.7, 43.2, 13.4. HRMS (ESI): $\mathrm{m} / z$ calcd for $\mathrm{C}_{18} \mathrm{H}_{18} \mathrm{Cl}_{2} \mathrm{~F}_{3} \mathrm{~N}_{5} \mathrm{O}_{2} \mathrm{~S}[\mathrm{M}+\mathrm{Na}]^{+}: 518.0408$, found 518.0407 .

\subsection{Synthesis of 2,6-Dichloro-N-(N-(4-(diethylamino)-6-(2,2,2-trifluoroethoxy)pyrimidin-2-yl)- carbamimidoyl)benzamide (32)}

Compound 32 was synthesized by the method described in [23]. A solution of compound 32a $(0.37 \mathrm{~g}, 0.75 \mathrm{mmol})$ and EDCI $(0.28 \mathrm{~g}, 1.49 \mathrm{mmol})$ was stirred at $0{ }^{\circ} \mathrm{C}$ for $30 \mathrm{~min}$ and hexamethyldisilazide (HMDS, $1.2 \mathrm{~g}, 7.4 \mathrm{mmol}$ ) was added. After the mixture was stirred for $3 \mathrm{~h}$ under $0{ }^{\circ} \mathrm{C}$, the mixture was raised to room temperature and stood for $24 \mathrm{~h}$. The organic layer was diluted with EtOAc $(20 \mathrm{~mL})$, washed twice with a saturated $\mathrm{NaHCO}_{3}$ solution $(20 \mathrm{~mL})$ and brine $(20 \mathrm{~mL})$, dried with anhydrous $\mathrm{Na}_{2} \mathrm{SO}_{4}$ and evaporated in vacuo. Finally, the residue was purified by silica gel column chromatography to afford compound 32 as a white solid, $\mathrm{mp} 166-168{ }^{\circ} \mathrm{C}$, yield $=81 \%$. ${ }^{1} \mathrm{H}-\mathrm{NMR}\left(\mathrm{DMSO}-d_{6}\right) \delta 11.25(\mathrm{~s}, 1 \mathrm{H}, \mathrm{NH}), 9.56(\mathrm{~s}, 1 \mathrm{H}, \mathrm{NH}), 9.48(\mathrm{~s}, 1 \mathrm{H}, \mathrm{NH}), 7.46(\mathrm{~d}, J=8.1 \mathrm{~Hz}, 2 \mathrm{H}, \mathrm{Ph})$, $7.35(\mathrm{dd}, J=8.8,7.5 \mathrm{~Hz}, 1 \mathrm{H}, \mathrm{Ph}), 5.85(\mathrm{~s}, 1 \mathrm{H}, \mathrm{ArH}), 4.98\left(\mathrm{q}, J=9.0 \mathrm{~Hz}, 2 \mathrm{H}, \mathrm{OCH}_{2} \mathrm{CF}_{3}\right), 3.69-3.40(\mathrm{~m}, 4 \mathrm{H}$, $\left.\mathrm{CH}_{2} \mathrm{CH}_{3}\right), 1.13\left(\mathrm{t}, J=7.0 \mathrm{~Hz}, 6 \mathrm{H}, \mathrm{CH}_{2} \mathrm{CH}_{3}\right) .{ }^{13} \mathrm{C}-\mathrm{NMR}$ (DMSO- $\left.d_{6}\right) \delta 176.3,169.0,163.2,160.1,157.5$, 140.6, 131.0, 130.6, 129.0, 128.9, 126.0, 123.7, 80.8, 62.7, 62.4, 62.2, 43.5, 13.6. HRMS (ESI): $\mathrm{m} / \mathrm{z}$ calcd for $\mathrm{C}_{18} \mathrm{H}_{19} \mathrm{Cl}_{2} \mathrm{~F}_{3} \mathrm{~N}_{6} \mathrm{O}_{2}[\mathrm{M}+\mathrm{Na}]^{+}:$501.0796, found 501.0791.

\subsection{Synthesis of the Target Compounds 33-38}

Compounds $\mathbf{3 3}-\mathbf{3 8}$ were prepared by a method similar to that used for compounds 1-30. 
5-Chloro-N-((4-chloro-6-(diethylamino)pyrimidin-2-yl)carbamoyl)-1-methyl-1H-pyrazole-4-carboxamide (33). White solid, mp 96-97 ${ }^{\circ} \mathrm{C}$, yield $=88 \% .{ }^{1} \mathrm{H}-\mathrm{NMR}\left(\mathrm{DMSO}-d_{6}\right) \delta 11.27(\mathrm{~s}, 1 \mathrm{H}, \mathrm{NH}), 10.73(\mathrm{~s}, 1 \mathrm{H}, \mathrm{NH})$, $8.30(\mathrm{~s}, 1 \mathrm{H}, \mathrm{ArH}), 6.50(\mathrm{~s}, 1 \mathrm{H}, \mathrm{ArH}), 3.86\left(\mathrm{~s}, 3 \mathrm{H}, \mathrm{N}-\mathrm{CH}_{3}\right), 3.43\left(\mathrm{~s}, 4 \mathrm{H}, \mathrm{CH}_{2} \mathrm{CH}_{3}\right), 1.11(\mathrm{t}, J=7.0 \mathrm{~Hz}, 6 \mathrm{H}$, $\mathrm{CH}_{2} \mathrm{CH}_{3}$ ). ${ }^{13} \mathrm{C}-\mathrm{NMR}\left(\mathrm{DMSO}-d_{6}\right) \delta 162.7,162.0,159.0,157.1,149.7,140.2,131.8,113.0,97.3,42.9,37.6$, 13.6. HRMS (ESI): $m / z$ calcd for $\mathrm{C}_{14} \mathrm{H}_{17} \mathrm{Cl}_{2} \mathrm{~N}_{7} \mathrm{O}_{2}[\mathrm{M}+\mathrm{H}]^{+}: 386.0899$, found 386.0929 .

N-((5-(sec-Butyl)-4-chloro-6-(diethylamino)pyrimidin-2-yl)carbamoyl)-5-chloro-1-methyl-1H-pyrazole-4-car boxamide (34). White solid, mp 159-161 ${ }^{\circ} \mathrm{C}$, yield $=75 \% .{ }^{1} \mathrm{H}-\mathrm{NMR}\left(\mathrm{DMSO}-d_{6}\right) \delta 11.10(\mathrm{~s}, 1 \mathrm{H}, \mathrm{NH}), 10.87$ (s, 1H, NH), $8.34(\mathrm{~s}, 1 \mathrm{H}, \mathrm{ArH}), 3.86\left(\mathrm{~s}, 3 \mathrm{H}, \mathrm{N}-\mathrm{CH}_{3}\right), 3.23\left(\mathrm{dq}, J=14.0,7.0 \mathrm{~Hz}, 2 \mathrm{H}, \mathrm{CHCH}_{2} \mathrm{CH}_{3}\right), 2.79$ (q, $J=7.3 \mathrm{~Hz}, 1 \mathrm{H}, \mathrm{CH}), 1.78\left(\mathrm{dtd}, J=15.2,13.6,7.3 \mathrm{~Hz}, 2 \mathrm{H}, \mathrm{CH}_{2} \mathrm{CH}_{3}\right), 1.39\left(\mathrm{~d}, J=7.2 \mathrm{~Hz}, 3 \mathrm{H}, \mathrm{CHCH}_{3}\right)$, $1.22\left(\mathrm{t}, J=2.8 \mathrm{~Hz}, 2 \mathrm{H}, \mathrm{CH}_{2} \mathrm{CH}_{3}\right), 1.15\left(\mathrm{t}, J=7.0 \mathrm{~Hz}, 6 \mathrm{H}, \mathrm{CH}_{2} \mathrm{CH}_{3}\right), 0.73\left(\mathrm{t}, J=7.4 \mathrm{~Hz}, 3 \mathrm{H}, \mathrm{CHCH}_{2} \mathrm{CH}_{3}\right)$. ${ }^{13}$ C-NMR (DMSO-d ${ }_{6}$ ) $\delta 169.4,162.3,158.9,153.5,149.7,140.2,131.9,119.1,112.7,45.7,37.5,35.2,27.9$, 18.4, 13.8, 13.4. HRMS (ESI): $m / z$ calcd for $\mathrm{C}_{18} \mathrm{H}_{25} \mathrm{Cl}_{2} \mathrm{~N}_{7} \mathrm{O}_{2}[\mathrm{M}+\mathrm{Na}]^{+}:$464.1344, found 464.1363.

5-Chloro-N-((4-chloro-6-(2,2,2-trifluoroethoxy)pyrimidin-2-yl)carbamoyl)-1-methyl-1H-pyrazole-4-carboxamide (35). White solid, mp 181-183 ${ }^{\circ} \mathrm{C}$, yield $=85 \% .{ }^{1} \mathrm{H}-\mathrm{NMR}\left(\mathrm{DMSO}-d_{6}\right) \delta 11.40(\mathrm{~s}, 1 \mathrm{H}, \mathrm{NH}), 11.10(\mathrm{~s}, 1 \mathrm{H}$, $\mathrm{NH}), 8.37$ (s, 1H, ArH), 7.07 (s, 1H, ArH), 5.11 (q, J = 8.9 Hz, 2H, OCH $\left.\mathrm{CF}_{3}\right), 3.86\left(\mathrm{~s}, 3 \mathrm{H}, \mathrm{N}-\mathrm{CH}_{3}\right)$. ${ }^{13}$ C-NMR (DMSO- $d_{6}$ ) $\delta 169.9,162.8,161.8,156.8,149.4,140.3,132.2,112.3,102.9,63.5,63.2,62.9,37.5$. HRMS (ESI): $m / z$ calcd for $\mathrm{C}_{12} \mathrm{H}_{9} \mathrm{Cl}_{2} \mathrm{~F}_{3} \mathrm{~N}_{6} \mathrm{O}_{3}[\mathrm{M}+\mathrm{H}]^{+}: 434.9963$, found 434.9975 .

N-((5-(sec-Butyl)-4,6-dichloropyrimidin-2-yl)carbamoyl)-2-chloronicotinamide (36). White solid, $\mathrm{mp}$ 139-140 ${ }^{\circ} \mathrm{C}$, yield = 86\%. ${ }^{1} \mathrm{H}-\mathrm{NMR}\left(\mathrm{DMSO}_{6}\right) \delta 11.49(\mathrm{~s}, 1 \mathrm{H}, \mathrm{NH}), 10.99(\mathrm{~s}, 1 \mathrm{H}, \mathrm{NH}), 8.55$ (dd, $J=4.9,1.9 \mathrm{~Hz}, 1 \mathrm{H}, \mathrm{ArH}), 8.09(\mathrm{dd}, J=7.6,1.9 \mathrm{~Hz}, 1 \mathrm{H}, \mathrm{ArH}), 7.58(\mathrm{dd}, J=7.6,4.8 \mathrm{~Hz}, 1 \mathrm{H}, \mathrm{ArH}), 3.40(\mathrm{dt}$, $J=9.0,7.1 \mathrm{~Hz}, 1 \mathrm{H}, \mathrm{CH}), 2.01-1.85\left(\mathrm{~m}, 1 \mathrm{H}, \mathrm{CHCH}_{2} \mathrm{CH}_{3}\right), 1.73\left(\mathrm{dt}, J=13.9,7.1 \mathrm{~Hz}, 1 \mathrm{H}, \mathrm{CHCH}_{2} \mathrm{CH}_{3}\right)$, $1.33\left(\mathrm{~d}, J=7.2 \mathrm{~Hz}, 3 \mathrm{H}, \mathrm{CHCH}_{3}\right), 0.81\left(\mathrm{t}, J=7.4 \mathrm{~Hz}, 3 \mathrm{H}, \mathrm{CHCH}_{2} \mathrm{CH}_{3}\right) .{ }^{13} \mathrm{C}-\mathrm{NMR}\left(\mathrm{DMSO}-d_{6}\right) \delta 167.9$, $154.5,152.1,148.9,146.8,139.1,132.4,129.8,124.0,37.1,27.1,18.2,13.3$. HRMS (ESI): $\mathrm{m} / z$ calcd for $\mathrm{C}_{15} \mathrm{H}_{14} \mathrm{Cl}_{3} \mathrm{~N}_{5} \mathrm{O}_{2}[\mathrm{M}+\mathrm{Na}]^{+}:$424.0111, found 424.0112 .

2-Chloro-N-((4-(diethylamino)-6-(2,2,2-trifluoroethoxy)pyrimidin-2-yl)carbamoyl)nicotinamide (37). White solid, mp 124-126 ${ }^{\circ} \mathrm{C}$, yield = 85\%. ${ }^{1} \mathrm{H}-\mathrm{NMR}\left(\mathrm{DMSO}-d_{6}\right) \delta 12.33(\mathrm{~s}, 1 \mathrm{H}, \mathrm{NH}), 10.30(\mathrm{~s}, 1 \mathrm{H}, \mathrm{NH}), 8.53$ $(\mathrm{dd}, J=4.9,1.9 \mathrm{~Hz}, 1 \mathrm{H}, \mathrm{ArH}), 8.06(\mathrm{dd}, J=7.6,1.9 \mathrm{~Hz}, 1 \mathrm{H}, \mathrm{ArH}), 7.55(\mathrm{dd}, J=7.6,4.9 \mathrm{~Hz}, 1 \mathrm{H}, \mathrm{ArH}), 5.84$ $(\mathrm{s}, 1 \mathrm{H}, \mathrm{ArH}), 4.97\left(\mathrm{q}, J=9.0 \mathrm{~Hz}, 2 \mathrm{H}, \mathrm{OCH}_{2} \mathrm{CF}_{3}\right), 3.47\left(\mathrm{~s}, 4 \mathrm{H}, \mathrm{CH}_{2} \mathrm{CH}_{3}\right), 1.11\left(\mathrm{t}, J=7.0 \mathrm{~Hz}, 6 \mathrm{H}, \mathrm{CH}_{2} \mathrm{CH}_{3}\right)$. ${ }^{13}$ C-NMR (DMSO-d ${ }_{6}$ ) $\delta$ 169.2, 167.3, 163.1, 156.7, 151.5, 146.5, 138.6, 133.5, 125.9, 124.0, 80.5, 62.6, 62.3, 62.1, 61.8, 43.4, 13.5. HRMS (ESI): $m / z$ calcd for $\mathrm{C}_{17} \mathrm{H}_{18} \mathrm{ClF}_{3} \mathrm{~N}_{6} \mathrm{O}_{3}[\mathrm{M}+\mathrm{Na}]^{+}:$469.0979, found 469.0966 .

1-(4-Chloro-6-(diethylamino)pyrimidin-2-yl)-3-(2,6-dichlorophenyl)urea (38). White solid, mp 98-99 ${ }^{\circ} \mathrm{C}$, yield $=78 \% .{ }^{1} \mathrm{H}-\mathrm{NMR}\left(\mathrm{DMSO}-\mathrm{d}_{6}\right) \delta 10.90(\mathrm{~s}, 1 \mathrm{H}, \mathrm{NH}), 10.11(\mathrm{~s}, 1 \mathrm{H}, \mathrm{NH}), 7.58(\mathrm{~d}, J=8.1 \mathrm{~Hz}, 2 \mathrm{H}, \mathrm{Ph})$, $7.37(\mathrm{t}, J=8.1 \mathrm{~Hz}, 1 \mathrm{H}, \mathrm{Ph}), 6.47(\mathrm{~s}, 1 \mathrm{H}, \mathrm{ArH}), 3.33\left(\mathrm{~s}, 4 \mathrm{H}, \mathrm{CH}_{2} \mathrm{CH}_{3}\right), 2.50\left(\mathrm{~s}, 6 \mathrm{H}, \mathrm{CH}_{2} \mathrm{CH}_{3}\right) .{ }^{13} \mathrm{C}-\mathrm{NMR}$ $\left(\right.$ DMSO- $\left.d_{6}\right) \delta 161.3,158.9,157.5,151.8,133.9,132.8,129.5,129.0,95.6,43.1,12.8$. HRMS (ESI): $\mathrm{m} / \mathrm{z}$ calcd for $\mathrm{C}_{15} \mathrm{H}_{16} \mathrm{Cl}_{3} \mathrm{~N}_{5} \mathrm{O}[\mathrm{M}+\mathrm{H}]^{+}:$388.0499, found 388.0488 .

\subsection{Insecticidal Biological Assay}

All bioassays were performed on representative test organisms reared in the laboratory. The bioassay was repeated at $25 \pm 1{ }^{\circ} \mathrm{C}$ according to statistical requirements. Assessments were made on a dead/alive basis and mortality rates were corrected using Abbott's formula [24]. Evaluations are based on a percentage scale of $0-100$ in which $0=$ no activity and $100=$ total kill.

\subsubsection{Toxicity against Mosquito (Culex pipiens pallens)}

The toxicities of compounds 1-25, 31 and 37 against mosquito were evaluated according to the reported procedure $[25,26]$. One milliliter of different concentrated dilutions of each compound was added to $99 \mathrm{~mL}$ of water to obtain different concentrations of tested solution. Then 20 fourth-instar mosquito larvae were put into the solution. Percentage mortalities were evaluated 8 day after treatment. 
For comparative purposes, fipronil was tested under the same conditions, and each test was performed in triplicate.

\subsubsection{Stomach Toxicity against Oriental Armyworm (Mythimna separata)}

The stomach toxicities of compounds 1-25, 31, 37 and the contrast fipronil against oriental armyworm were evaluated by foliar application using the reported procedure [27]. For the foliar armyworm tests, individual corn leaves were placed on moistened pieces of filter paper in Petri dishes. The leaves were then sprayed with the test solution and allowed to dry. The dishes were infested with 10 fourth-instar Oriental armyworm larvae. Percentage mortalities were evaluated 3 days after treatment. Each treatment was performed three times

\subsection{In Vitro Antifungal Bioassay}

The antifungal activities were screened and evaluated by the poison plate technique [28]. All final compounds were dissolved in DMF $(0.1 \mathrm{~mL})$ before mixing with potato dextrose agar (PDA; $9.9 \mathrm{~mL})$. The compounds were tested at a concentration of $50 \mu \mathrm{g} \mathrm{mL}{ }^{-1}$. All fungi were cultivated in PDA at $27 \pm 1^{\circ} \mathrm{C}$ for 4 days to make new mycelium for the identification of antifungal activity. Then, mycelia dishes of approximately $5 \mathrm{~mm}$ diameter were cut from the culture medium. A mycelium was obtained using a germ-free inoculation needle and inoculated in the middle of the PDA plate aseptically. The inoculated plates were incubated at $27 \pm 1{ }^{\circ} \mathrm{C}$ for 5 days. DMF in sterile distilled water served as the negative control, whereas hymexazol served as the positive control. Each treatment condition consisted of three replicates. Radial growth of the fungal colonies was measured, and the data were statistically analyzed. Inhibitory effects of the test compounds in vitro on these fungi were calculated by the formula $\mathrm{I}(\%)=[(C-T) /(C-0.5)] \times 100$, where $C$ represents the diameter of fungal growth on untreated PDA, $T$ represents the diameter of fungi on treated PDA, and $I$ represents the inhibition rate.

\subsection{In Vivo Antifungal Bioassay against Sclerotinia sclerotiorum}

The requisite amounts of compounds 19 and 25 was disolved in sterile Tween $80(0.1 \%, v / v)$ solution to give different concentration test solution $\left(500,1500\right.$ and $\left.3000 \mu \mathrm{g} \mathrm{mL}{ }^{-1}\right)$. For protective activity assay [29], fresh leaves were sprayed with these solution (10 mL for each leave) until liquid flowed on surface at $24 \mathrm{~h}$ before inoculation. A colonized mycelial plug (5 $\mathrm{mm}$ in diameter) from a 5-day-old PDA culture of carbendazim-resistant Sclerotinia sclerotiorum was placed on the surface of fresh leaves. Inoculated leaves were placed at $25{ }^{\circ} \mathrm{C}$ with $80 \%$ relative humidity for disease development. After 5 days, the average lesion diameter was determined by measuring each lesion in two perpendicular directions. The lengths of the long and short axes were averaged and disease control efficacy was calculated as follows: disease control efficacy $(\%)=($ lesion diameter in the water control-lesion diameter in the treatment)/lesion diameter in the water control $\times 100$ [30].

\subsection{Zebrafish Embryo Toxicity Assay}

Zebrafish wild-type $\mathrm{AB}$ (Danio rerio $A B$ ) was introduced from the National Zebrafish Center (Wuhan, China). The zebrafish for experiment was cultured by the five-layer zebrafish culture system of Beijing Aisheng Technology Development Co., Ltd. (Beijing, China) to the fifth generation. Animals were housed in feeding system $\left(\mathrm{pH}\right.$ 6.5-7.5, oxygen content $>85 \%$, water temperture $28^{\circ} \mathrm{C}$, conductivity $500 \pm 50 \mu \mathrm{s} \mathrm{cm}^{-1}$, Light-dark ratio $14 \mathrm{~h} / 10 \mathrm{~h}$ ). Natural mortality during feeding was less than $1 \%$. Fish were fed twice a day with Artemia salina, the total weight of feeding was $5 \pm 1 \%$ of zebrafish body weight. For this experiment, a sexually mature 8-month zebrafish were used. Males and females were maintained separately until the night before the spawning at a ratio of 1:2 and the light-dark ratio was controlled at $14 \mathrm{~h} / 10 \mathrm{~h}$. Embryos were obtained by naturally mate [31].

Toxicological analyses were based on approved standard OECD TG 236: Fish Embryo Toxicity (FET) Test [32]. 20 embryos were distributed in each of different concentrations of the compounds 
to be analyzed, a positive control (3,4-dichloroaniline), a negative control (water) and a solvent control (10\% Hank's solution). All of the treatments were cared at $26^{\circ} \mathrm{C} \pm 1{ }^{\circ} \mathrm{C}$, light/dark cycle of 14/10 h. The entire experiment was carried out for $96 \mathrm{~h}$. All of the data were analyzed by SPSS 19.0 (IBM Corporation, New York, NY, USA), LC 50 was corrected taking into account control mortality with Abbott's formula [15].

\section{Conclusions}

In summary, a series of novel benzoylpyrimidinylurea derivatives were designed and synthesized. Although we did not find any compounds which possessed both insecticidal and antifungal as efficient as we expected, compounds 7 and 25 still exhibited excellent larvicidal activity against mosquito (Culex pipiens pallens) and broad-spectrum antifungal activity against fourteen phytopathogenic fungi, respectively. High efficiency, low toxicity and environmentally friendly pesticides are consistent with the requirements of sustainable agricultural development. Therefore, compounds 7 and 25 with low toxicity to zebrafish will be potential lead compounds to develop green mosquitocides and broad-spectrum fungicides.

Supplementary Materials: The following are available online. Figures S1-S101.

Author Contributions: R.S. conceived and designed the experiments; P.C., X.S. and W.K. performed the experiments, analyzed the data; X.S. drafted the manuscript; R.S. revised the manuscript; Y.F. and H.Z. gave some suggestion to the experiment.; P.C and X.S contributed equally to this work. All authors have read and approved the final manuscript.

Funding: This work was financially supported by the National Science Foundation of China (No. 21462028), Startup Foundation for Outstanding Young Scientists of Hainan University (kyqd1640) and Hainan Province Natural Science Foundation of China (No. 20163040).

Conflicts of Interest: The authors declare no conflict of interest.

\section{References}

1. Ishaaya, I.; Degheele, D. Insecticides with Novel Modes of Action: Mechanisms and Application; Springer: Berlin/Heidelberg, Germany, 1998; p. 289, ISBN 9783540630586.

2. Oberlander, H.; Silhacek, D.L. Mode of action of insect growth regulators in lepidopteran tissue culture. Pestic. Sci. 1998, 54, 300-302. [CrossRef]

3. Sun, R.F.; Liu, C.J.; Zhang, H.; Wang, Q.M. Benzoylurea Chitin Synthesis Inhibitors. J. Agric. Food Chem. 2015, 63, 6847-6865. [CrossRef] [PubMed]

4. Sun, R.F.; Lu, M.Y.; Chen, L.; Li, Q.S.; Song, H.B.; Bi, F.C.; Huang, R.Q.; Wang, Q.M. Design, synthesis, bioactivity, and structure-activity relationship (SAR) studies of novel benzoylphenylureas containing oxime ether group. J. Agric. Food Chem. 2008, 56, 11376-11391. [CrossRef] [PubMed]

5. Sun, R.F.; Li, Y.Q.; Lu, M.Y.; Xiong, L.X.; Wang, Q.M. Synthesis, larvicidal activity, and SAR studies of new benzoylphenylureas containing oxime ether and oxime ester group. Bioorg. Med. Chem. Lett. 2010, 20, 4693-4699. [CrossRef] [PubMed]

6. Sun, R.F.; Zhang, Y.L.; Bi, F.C.; Wang, Q.M. Design, synthesis, and bioactivity study of novel benzoylpyridazyl ureas. J. Agric. Food Chem. 2009, 57, 6356-6361. [CrossRef] [PubMed]

7. Sun, R.F.; Li, Y.Q.; Xiong, L.X.; Liu, Y.X.; Wang, Q.M. Design, synthesis, and insecticidal evaluation of new benzoylureas containing isoxazoline and isoxazole group. J. Agric. Food Chem. 2011, 59, 4851-4859. [CrossRef] [PubMed]

8. Sun, R.F.; Wang, Z.W.; Li, Y.Q.; Xiong, L.X.; Liu, Y.X.; Wang, Q.M. Design, synthesis, and insecticidal evaluation of new benzoylureas containing amide and sulfonate groups based on the sulfonylurea receptor protein binding site for diflubenzuron and glibenclamide. J. Agric. Food Chem. 2013, 61, 517-522. [CrossRef] [PubMed]

9. Niyaz, N.M.; Hunter, R.; Johnson, T.C.; Trullinger, T.K.; Brown, A.V.; Bryan, K. Preparation of Substituted $N$-azinyl-N'-aryl Ureas as Insecticides. U.S. Patent 20090093486A1, 9 April 2009.

10. Gewehr, M. Fungicidal Mixtures Comprising Quinoline Fungicides. WO2018050421A1, 22 March 2018. 
11. Eberlin, A.R.; Frampton, C.S. A metastable polymorphic form of the antifungal anilinopyrimidine active pyrimethanil. Acta Crystallogr. Sect. E Crystallogr. Commun. 2017, 73, 886-889. [CrossRef] [PubMed]

12. Li, Y.; Liu, S.; Qin, Y.; Xing, R.; Chen, X.; Li, K.; Li, P. Synthesis of novel pyrimethanil grafted chitosan derivatives with enhanced antifungal activity. BioMed Res. Int. 2016, 2016, 8196960. [CrossRef] [PubMed]

13. Choi, W.S.; Nam, S.W.; Ahn, E.K.; Park, B.S.; Lee, S.E.; Kim, T.J.; Choi, I.Y. Synthesis and fungicidal activity of $N$-[4-(4-fluoro)phenyl-2-piperidin-4-yl-thiazol-5-yl]pyrimidin-2-yl-N-phenylamines on Phytophthora capsici. J. Korean Soc. Appl. Biol. Chem. 2010, 53, 206-214. [CrossRef]

14. Nam, S.W.; Choi, I.Y.; Choi, W.S. Synthesis and antifungal activity of 5-[2-(Alkylamino)pyrimidin-4-yl]-4phenylthiazol-2-cycloalkylamine derivatives on Phytophthora capsici. J. Korean Soc. Appl. Biol. Chem. 2011, 54, 395-402. [CrossRef]

15. Fan, Y.M.; Feng, Q.; Lai, K.H.; Huang, W.K.; Zhang, C.H.; Li, Q.X. Toxic effects of indoxacarb enantiomers on the embryonic development and induction of apoptosis in zebrafish larvae (Danio rerio). Environ. Toxicol. 2017, 32, 7-16. [CrossRef] [PubMed]

16. Fan, Y.M.; Miao, W.G.; Lai, K.H.; Huang, W.K.; Song, R.X.; Li, Q.X. Developmental toxicity and inhibition of the fungicide hymexazol to melanin biosynthesis in zebrafish embryos. Pestic. Biochem. Phys. 2018, 147, 139-144. [CrossRef] [PubMed]

17. Zhang, J.; Jin, G.Y.; Xiao, S.; Wu, J.; Cao, S. Novel synthesis of 1,4,5-trisubstituted 1,2,3-triazoles via a one-pot three-component reaction of boronic acids, azide, and active methylene ketones. Tetrahedron 2013, 69, 2352-2356. [CrossRef]

18. Sreedhar, B.; Arundhathi, R.; Reddy, P.L.; Kantam, M.L. CuI Nanoparticles for C-N and C-O Cross Coupling of Heterocyclic Amines and Phenols with Chlorobenzenes. J. Org. Chem. 2009, 74, 7951-7954. [CrossRef] [PubMed]

19. Jansa, P.; Holy, A.; Dracinsky, M.; Kolman, V.; Janeba, Z.; Kostecka, P.; Kmonickova, E.; Zidek, Z. 5-Substituted 2-amino-4,6-dihydroxypyrimidines and 2-amino-4,6-dichloropyrimidines. Synthesis and inhibitory effects on immune-activated nitric oxide production. Med. Chem. Res. 2014, 23, 4482-4490. [CrossRef]

20. He, H.F.; Wang, X.Y.; Shi, L.Q.; Yin, W.Y.; Yang, Z.W.; He, H.W.; Liang, Y. Synthesis, antitumor activity and mechanism of action of novel 1,3-thiazole derivatives containing hydrazide-hydrazone and carboxamide moiety. Bioorg. Med. Chem. Lett. 2016, 26, 3263-3270. [CrossRef] [PubMed]

21. Lei, P.; Xu, Y.; Du, J.; Yang, X.L.; Yuan, H.Z.; Xu, G.F.; Ling, Y. Design, synthesis and fungicidal activity of N-substituted benzoyl-1,2,3,4-tetrahydroquinolyl-1-carboxamide. Bioorg. Med. Chem. Lett. 2016, 26, 2544-2546. [CrossRef] [PubMed]

22. Mawnai, I.L.; Adhikari, S.; Kaminsky, W.; Kollipara, M.R. Synthesis of strained complexes of arene $\mathrm{d}^{6}$ metals with benzoylthiourea and their spectral studies. J. Organomet. Chem. 2018, 869, 26-36. [CrossRef]

23. Shinada, T.; Umezawa, T.; Ando, T.; Kozuma, H.; Ohfune, Y. A new entry for the synthesis of $N$-acyl- $N^{\prime}-$ substituted guanidines. Tetrahedron Lett. 2006, 47, 1945-1947. [CrossRef]

24. Abbott, W.S. A method of computing the effectiveness of an insecticide. J. Am. Mosq. Control Assoc. 1987, 3, 302-303. [CrossRef] [PubMed]

25. Zhao, Q.Q.; Li, Y.Q.; Xiong, L.X.; Wang, Q.M. Design, Synthesis and Insecticidal Activity of Novel Phenylpyrazoles Containing a 2,2,2-Trichloro-1-alkoxyethyl Moiety. J. Agric. Food Chem. 2010, 58, 4992-4998. [CrossRef] [PubMed]

26. Labbe, P.; Berticat, C.; Berthomieu, A.; Unal, S.; Bernard, C.; Weill, M.; Lenormand, T. Forty years of erratic insecticide resistance evolution in the mosquito Culex pipiens. PLoS Genet. 2007, 3, 2190-2199. [CrossRef] [PubMed]

27. Zhao, Q.Q.; Shang, J.; Liu, Y.X.; Wang, K.Y.; Bi, F.C.; Huang, R.Q.; Wang, Q.M. Synthesis and insecticidal activities of novel $N$-sulfenyl- $N^{\prime}$-tert-butyl- $N, N^{\prime}$-diacylhydrazines. 1 . $\mathrm{N}$-alkoxysulfenate derivatives. J. Agric. Food Chem. 2007, 55, 9614-9619. [CrossRef] [PubMed]

28. Song, X.M.; Liu, C.J.; Chen, P.Q.; Zhang, H.; Sun, R.F. Natural Product-Based Pesticide Discovery: Design, Synthesis and Bioactivity Studies of N-Amino-Maleimide Derivatives. Molecules 2018, 23, 1521. [CrossRef] [PubMed]

29. Hou, Y.; Mao, X.; Lin, S.; Song, X.; Duan, Y.; Wang, J.; Zhou, M. Activity of a novel succinate dehydrogenase inhibitor fungicide pyraziflumid against Sclerotinia sclerotiorum. Pestic. Biochem. Phys. 2018, 145, $22-28$. [CrossRef] [PubMed] 
30. Kuang, J.; Hou, Y.P.; Wang, J.X.; Zhou, M.G. Sensitivity of Sclerotinia sclerotiorum to fludioxonil: In vitro determination of baseline sensitivity and resistance risk. Crop Prot. 2011, 30, 876-882. [CrossRef]

31. Bridi, D.; Altenhofen, S.; Gonzalez, J.B.; Reolon, G.K.; Bonan, C.D. Glyphosate and Roundup ${ }^{\circledR}$ alter morphology and behavior in zebrafish. Toxicology 2017, 392, 32-39. [CrossRef] [PubMed]

32. Kluever, N.; Vogs, C.; Altenburger, R.; Escher, B.I.; Scholz, S. Development of a general baseline toxicity QSAR model for the fish embryo acute toxicity test. Chemosphere 2016, 164, 164-173. [CrossRef] [PubMed]

Sample Availability: Samples of the compounds are not available from the authors.

(C) 2018 by the authors. Licensee MDPI, Basel, Switzerland. This article is an open access article distributed under the terms and conditions of the Creative Commons Attribution (CC BY) license (http:/ / creativecommons.org/licenses/by/4.0/). 